\title{
Floristic diversity of two crystalline rocky outcrops in the Brazilian northeast semi-arid region ${ }^{1}$
}

\author{
POLYHANNA GOMES ${ }^{2,3}$ and MARCCUS ALVES 2
}

(received: March 19, 2010; accepted: October 21, 2010)

\begin{abstract}
Floristic diversity of two crystalline rocky outcrops in the Brazilian northeast semi-arid region). Floristic composition and structure of vegetation were studied in two rocky outcrop areas in the semi-arid region of northeastern Brazil. From April 2007 to September 2008, 18 monthly field trips were carried out. Vascular plants were randomly collected throughout the outcrop areas. For structural analysis, 30 plots of $1 \times 1 \mathrm{~m}$ were set in the vegetation islands. The checklist presented combines 211 species (69 families and 168 genera), although only 56 species were collected in the plots. Fabaceae (18 spp.; 8.5\%), Asteraceae (17 spp.; 8\%), Orchidaceae (13 spp.; 6.1\%), Euphorbiaceae (13 spp.; 6.1\%), Bromeliaceae (10 spp.; 4.7\%), and Poaceae (eight spp.; 3.8\%) are the richest families. Overall, 1,792 shrub and herbaceous specimens were counted in the plots. The Shannon-Wiener $(\mathrm{H})$ diversity index values were 2.572 and 2.547 nats individual ${ }^{-1}$. The species that presented the highest absolute abundance values (number of plants) had low frequencies in the plots and vice-versa. The biological spectrum had a high proportion of phanerophytes and therophytes, followed by cryptophytes, chamaephytes, and hemicryptophytes. The studied flora shares floristic components similar to other rocky outcrop areas of the semi-arid region in northeastern Brazil, including in relation to dominant groups in the vegetation structure.
\end{abstract}

Key words - Caatinga, inselbergs, life-forms, saxicolous plants

RESUMO - (Diversidade florística de dois afloramentos rochosos cristalinos no semi-árido, nordeste do Brasil). Composição florística e estrutura da vegetação foram estudadas em dois afloramentos rochosos localizados no semi-árido do nordeste do Brasil. Foram realizadas 18 excursões mensais, de abril de 2007 a setembro de 2008. Plantas vasculares foram coletadas aleatoriamente, no afloramento como um todo. Para análise estrutural foram plotadas 30 parcelas de $1 \times 1 \mathrm{~m}$ nas ilhas de vegetação. Foram encontradas 211 espécies (69 famílias e 168 gêneros), entretanto somente 56 espécies foram coletadas nas parcelas. As famílias com maior número de espécies foram Fabaceae (18 spp.; 9\%), Asteraceae (17 spp.; 8,5\%), Orchidaceae (13 spp.; 6,5\%), Euphorbiaceae (13 spp.; 6,5\%), Bromeliaceae (10 spp.; 5\%) e Poaceae (oito spp.; 4\%). Ao todo, foram contabilizados, nas parcelas, 1.792 indivíduos herbáceos e arbustivos. Os valores do índice de diversidade de Shannon-Wiener (H) foram de 2,572 e 2,547 nats ind. ${ }^{-1}$. As espécies que apresentaram maiores densidade apresentaram baixa freqüência nas parcelas e vice-versa. $\mathrm{O}$ espectro biológico apresentou alta proporção de fanerófitos e terófitos, seguidos por criptófitos, caméfitos e hemi-criptófitos. A flora estudada compartilha conjunto florístico semelhante a outros afloramentos rochosos do Nordeste do Brasil, inclusive em termos de grupos dominantes na estrutura da vegetação.

Palavras-chave - Caatinga, formas de vida, inselbergues, plantas saxícolas

\section{Introduction}

Few vegetation formations have caught as much attention from the scientific community as the rocky outcrops. Most of such interest is due to their wide distribution and because they represent good experimental models, especially due to their easily delimitable spatial boundaries (Porembski \& Barthlott

1. Part of the first author's MSc Dissertation, Programa de Pós Graduação em Biologia Vegetal of Universidade Federal de Pernambuco, PE, Brazil.

2. Universidade Federal de Pernambuco (UFPE), Centro de Ciências Biológicas, Laboratório de Morfo-Taxonomia Vegetal. Rua Professor Nelson Chaves, s/n, Cidade Universitária, Recife, PE, CEP 50372-970 Brazil.

3. Corresponding author: polyhannagomes@hotmail.com
2000). However, the interests primarily turn to those environments because they shelter a peculiar biota, with several endemic species (Oosting \& Anderson 1939, Porembski \& Barthlott 2000).

Studies in tropical rocky outcrop areas have been intensified since the end of last century. Most of them are from Porembski and collaborators (Porembski \& Barthlott 1997, Porembski et al. 1997, Porembski 2000, Porembski \& Barthlott 2000, among others).

Despite that, there is a great knowledge gap concerning the crystalline rocky outcrops in Northeastern Brazil (Martinelli 2007). In this region, they occur mainly in the Sertaneja Depression and the Borborema Plateau, as residual massifs or mountains (Silva et al. 1993), differing geologically and biogeographically from campos rupestres of Chapada Diamantina, 
mainly in metamorphic rocks from sedimentary origin (predominantly quartzites) (Silva et al. 1993, Alves \& Kolbek 1994, Alves et al. 2007).

The floristic inventories in northeastern crystalline rocky outcrop areas started with França et al. (1997) in the state of Bahia. Those authors also approached aspects of the vegetation's structure (França et al. 2005, 2006). After those initial studies, floristic checklists of rocky areas were published by Porto et al. (2008) for the state of Paraiba, Araújo et al. (2008) for the state of Ceará and finally Gomes \& Alves (2009) for the state of Pernambuco (figure 1).

Nevertheless, basic aspects of the vegetation, such as which groups are dominant, are not yet clear (Martinelli 2007), especially because the already existing work covers little of the quantity and diversity of the rocky outcrops found in the semi-arid region of Brazil (Ab’Sáber 2003). Thus, this study presents the floristic diversity of two rocky outcrops from crystalline origin located in the semi-arid region of the state of Pernambuco.

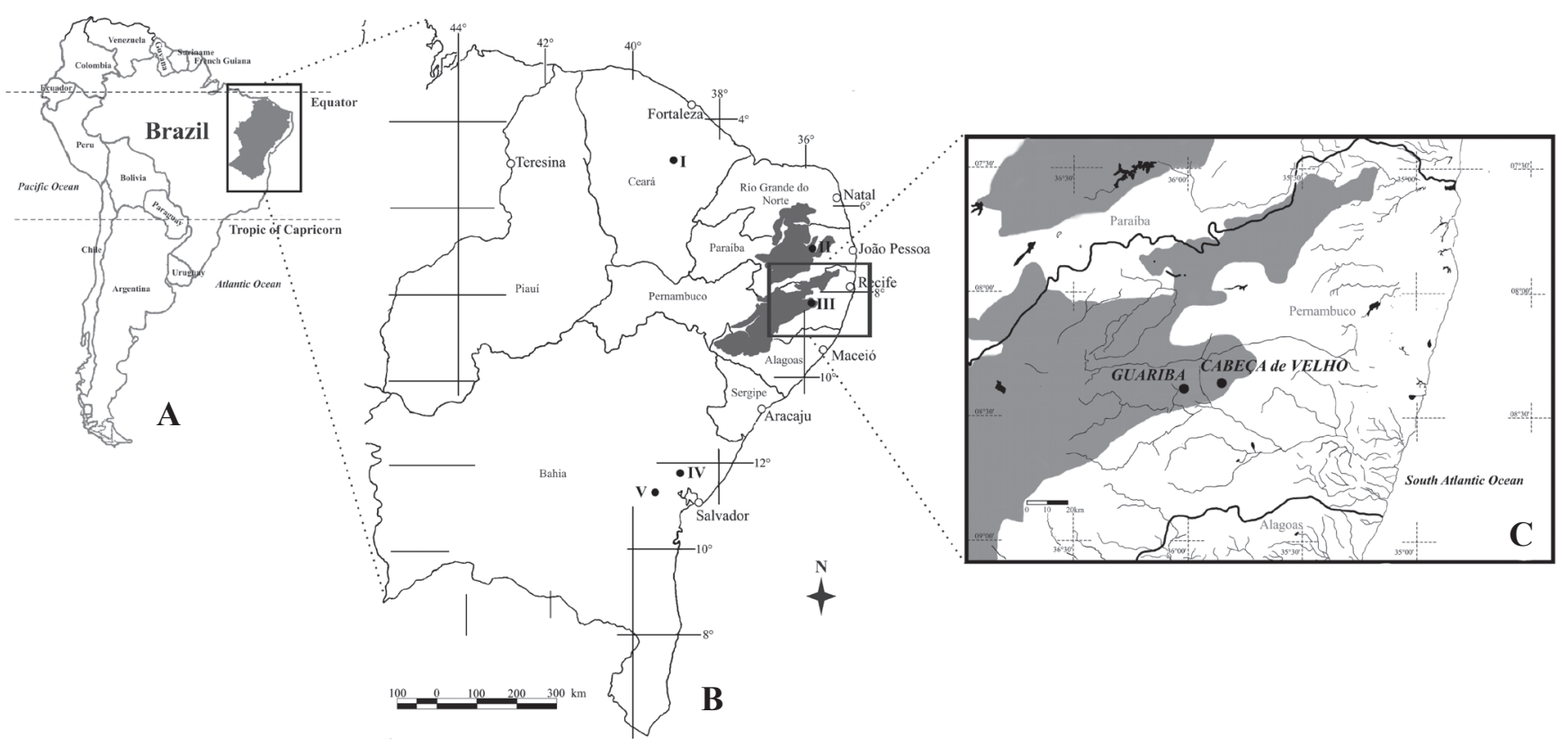

Figure 1. Location of study areas. Dark areas correspond to: A. Brazilian semi-arid; and (B and C) Borborema Plateau. B. Roman numerals are the floristic studies in northeastern crystalline rocky outcrop areas: (I) Araújo et al. (2008), (II) Porto et al. (2008), (III) Gomes \& Alves (2009), (IV, V) França et al. (1997, 2005, 2006).

\section{Material and methods}

Study area - Most part of the Northeastern region of Brazil is characterized by a semi-arid climate, seasonally dry and with very high temperatures (Andrade-Lima 1981). Sertaneja Depression is the typical landscape unit (around $370,000 \mathrm{~km}^{2}$ ) where the rainy season is limited to few months of the year and the temperature is very high (Silva et al. 1993). The annual pluviometry, in its core portion, is lesser than $600 \mathrm{~mm}$, with annual average temperature between $25-29{ }^{\circ} \mathrm{C}$ (Ab'Saber 1974, 2003, Silva et al. 1993).

Amongst the other landscape entities is the Borborema Plateau (around 45,000 $\mathrm{km}^{2}$ ), located in the extreme east of the semi-arid region (figure 1). It is formed from a series of strongly weathered crystalline massifs with an altitude of 600 to $1,000 \mathrm{~m}$ (Silva et al. 1993). The climate is characterized by accentuated irregularity in rain distribution and higher precipitation volume in the west than in the east (Silva et al. 1993). Despite having a seasonal climate as well, the climatic conditions are milder in the plateau than in the depressions between plateaus (Silva et al. 1993, Ab'Sáber 2003). The annual average precipitation values range from 600 to $1,000 \mathrm{~mm}$, which is higher than in the surrounding area (Conti 2005, ITEP 2010). However the average temperature is similar (Conti 2005, ITEP 2010).

The main vegetation is the savanna-steppe - caatinga (Silva et al. 1993). However, exceptional aspects of lithology, hydrology, topography, and paleobotany explain the existence of other vegetation types, such as humid forests (Ab'Sáber 2003). These humid forest refuges occurring in the Borborema Plateau are known locally as Brejos de Altitude and have a peculiar climate condition generated by the relief (see Vasconcelos-Sobrinho 1949, Andrade-Lima 1982). Thus, through these barriers to the air masses, the humidity is deposited in the windward 
strands, grottoes and mountain valleys (Andrade-Lima 1982). The vegetation established in these areas is ombrophilous or seasonal (Rodal et al. 2005) and can be classified as sub-montane when located between 100 and $600 \mathrm{~m}$ and montane when located over $600 \mathrm{~m}$ (Veloso et al. 1991).

The two rocky outcrop areas studied in this work are mountain tops inserted in a Forest refuges, called "Brejo de Agrestina" (Vasconcelos-Sobrinho 1949). They are $2 \mathrm{~km}$ apart from each other and approximately $150 \mathrm{~km}$ away from the Atlantic coast, in the state of Pernambuco (figure 1). The first one is called "Pedra da Guariba" $\left(08^{\circ} 22^{\prime} 55^{\prime}\right.$ " S-35 $50^{\circ}$ ' $38^{\prime} \mathrm{W}$; figures 2 and 3), with an altitude of $620 \mathrm{~m}$ and $c a$. $0.4 \mathrm{~km}^{2}$ in size. The second one is "Pedra Cabeça de Velho" $\left(08^{\circ} 23^{\prime} 29^{\prime \prime} \mathrm{S}-36^{\circ} 00^{\prime} 37^{\prime}\right.$ ' W; figures 4 and 5), which has an altitude of $740 \mathrm{~m}$ and $c a .0 .35 \mathrm{~km}^{2}$ in size. Both have a granite base with some gneiss inclusions.

In the study area, the dry season extends for 6 to 8 months, with precipitation between $12-52 \mathrm{~mm}$ per month, while the rainy season lasts for 4 to 5 months, with precipitation between 81-98 mm per month (ITEP 2010). The annual average for precipitation is $662 \mathrm{~mm}$ and for temperature is $22.5^{\circ} \mathrm{C}$, with a range of 20 to $24^{\circ} \mathrm{C}$ (ITEP 2010).

The surrounding areas are occupied by small and medium farms. Bovine and poultry livestock and subsistence agriculture are the main activities. The rocky outcrop areas studied are less degraded than their surroundings due to their low potential for agriculture. However, Cabeça de Velho was affected twice by accidental fires within a three-year period. There is no record of fires in Guariba, but this fragment suffers more pressure from visitors than the first one. The area is cited as of extreme importance for environment inventories and for the creation of a protection area (Martinelli 2007, MMA 2007).

Sampling - From April 2007 to September 2008, 18 monthly fieldtrips were made, totalizing $c a .210 \mathrm{~h}$ of sampling effort. Fertile specimens of vascular plants were randomly collected. The material was processed according to the usual methodology for botanical studies and was deposited in UFP herbarium, with duplicates in NY, RB, HUEFS, and IPA herbaria (see Thiers 2010).
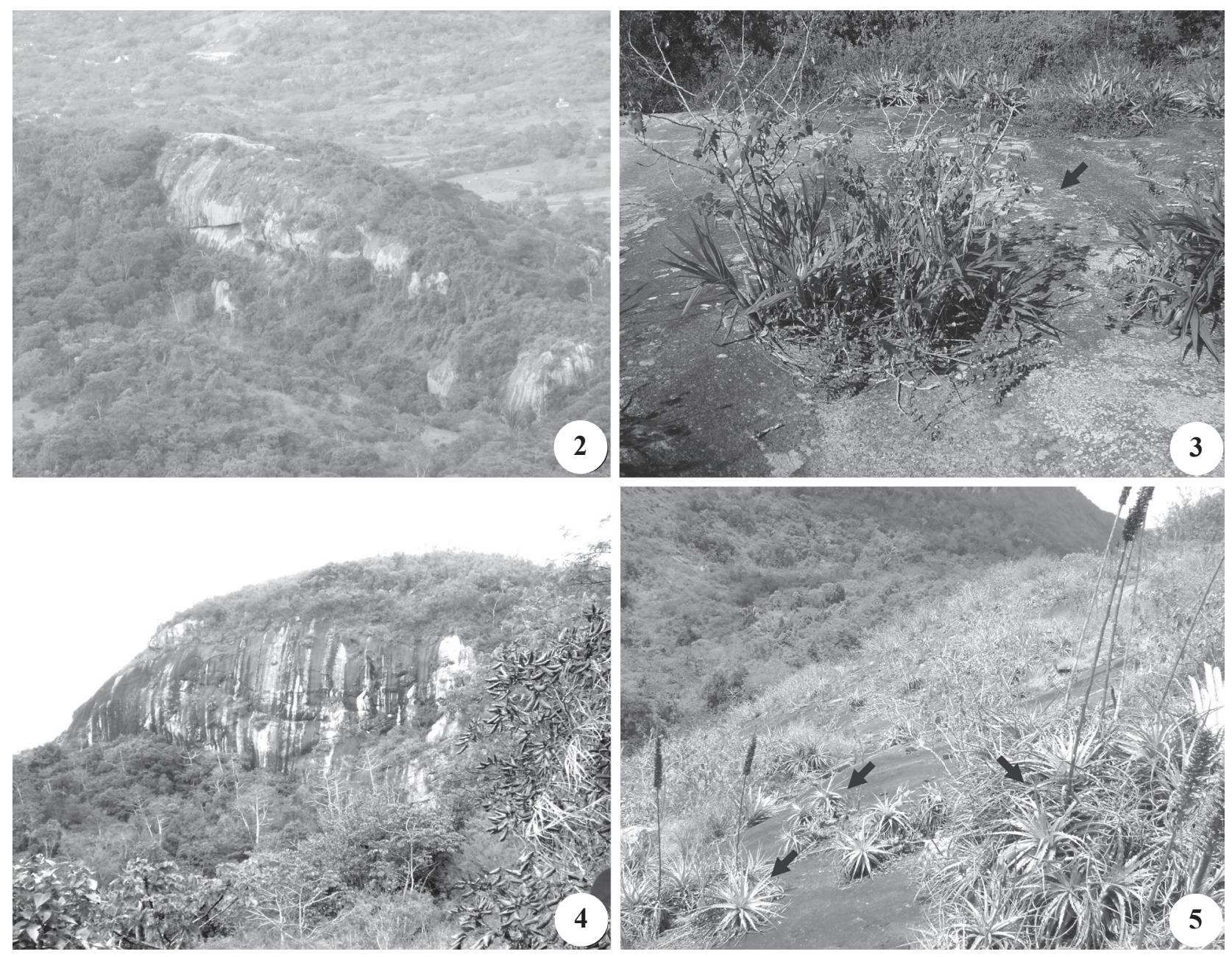

Figures 2-5. General aspect of two crystalline rocky outcrops in the Brazilian northeast semi-arid region: (2 and 3) Pedra da Guariba and (4 and 5) Pedra Cabeça de Velho. Arrows correspond to (2 and 4) island of vegetation. 
In rock outcrops there are extensive rock patches devoid of vascular vegetation (Meirelles et al. 1999). Due to this, in order to avoid some plots falling out on a bare surface (e.g. see Caiafa \& Silva 2007), our sampling unity for abundance and frequency analyses of the species were the vegetation islands (figures 2 and 5). All vegetation islands that were accessible on the eastern faces (windward) were numbered. The vegetation located leeward could not be included in the analyses because these sites were inaccessible due to their high slope. In total, 178 islands were marked: 109 in Guariba and 69 in Cabeça de Velho. Then, 30 vegetation islands were randomly chosen, 15 from each outcrop, and plots of $1 \times 1 \mathrm{~m}$ were plotted in each selected island. For the vegetation islands longer than $1 \mathrm{~m}$ length and/or width, the place to set the plot was randomly chosen, from left to right. In each plot the species and the number of individuals per species were counted. An individual was defined as being the whole aerial axis of a plant without any connection to another axis at or above soil level. For the plot analyses, the data were collected in July 2007 and 2008, since this is one of the months with the highest precipitation in the study locality.

Data analysis - Species identifications were made by reference to the collections available at UFP, PEUFR, IPA, HUEFS, SP and SPF, literature and taxonomists. For some taxa collected in a seedling stage in the plots, the species level identification was not possible. The floristic checklist is according to APG III (2009) and Tryon \& Tryon (1982). The species were classified into Raunkiaer's life-forms (1934). With the data from the plots, the parameters of absolute dominance, relative dominance, absolute frequency, and relative frequency were calculated according to Mueller-Dombois \& Ellenberg (1974). The Shannon-Wiener $(\mathrm{H})$ diversity and Jaccard similarity indexes were calculated with PAST software version $1.77^{\circ}$.

\section{Results}

Floristic - The 211 species were found in the random sampling collected from both localities and the whole outcrop area, consisting of 205 angiosperms and 6 pteridophytes, belonging to 69 families and 168 genera (table 1). The richest families were Fabaceae (18 spp.; 8.5\%), Asteraceae (17 spp.; 8\%), Orchidaceae (13 spp.; 6.2\%), Euphorbiaceae (12 spp.; 5.7\%), Bromeliaceae (10 spp.; 4.7\%), and Poaceae (eight spp.; 3.8\%), representing together $c a .35 \%$ of the taxa. The richest genera were Ipomoea, with four species, followed by Erythroxylum, Mandevilla, Tillandsia, Croton, Senna, and Hyptis, with three species each.

Overall, there were 143 species exclusive to one of the two areas and 67 shared species. The richness of Guariba (154 species) was greater than that of Cabeça de Velho (125 spp.). Guariba had 86 exclusive species and 18 exclusive families. In Cabeça de Velho, there were 57 exclusive species and 12 exclusive families (table 1 ). The Jaccard similarity index between the studied areas was 0.347 .

Table 1. Checklist of vascular plants, with localities (a), vouchers (b) and life-forms (c), collected in two rock outcrops of the semi-arid region in Brazil. (Gua = Pedra do Guariba; $\mathrm{Cab}=$ Pedra Cabeça de Velho; BSA = B.S. Amorim et al.; $\mathrm{EC}=\mathrm{E}$. Córdula et al.; JO = J. Oliveira et al.; JRM = J.R. Maciel et al.; $\mathrm{KM}=\mathrm{K}$. Mendes et al.; $\mathrm{KP}=\mathrm{K}$. Pinheiro et al.; $\mathrm{PG}=\mathrm{P}$. Gomes et al.; $\mathrm{Cr}=$ cryptophyte; $\mathrm{HCr}=$ hemicryptophyte; $\mathrm{Ch}=$ chamaephyte; $\mathrm{Ph}=$ phanerophyte; $\mathrm{Th}=$ therophyte. ${ }^{\mathrm{d} S p e c i e s}$ also found in the plots.)

\begin{tabular}{|c|c|c|c|c|c|}
\hline \multirow{2}{*}{ Family } & \multirow{2}{*}{ Species } & \multicolumn{2}{|c|}{ Locality $^{\mathrm{a}}$} & \multirow{2}{*}{ - Voucher ${ }^{\mathrm{b}}$} & \multirow{2}{*}{ Life-forms } \\
\hline & & Gua & $\mathrm{Cab}$ & & \\
\hline ACANTHACEAE & Dyschoriste maranhonis Kuntze & $\mathrm{X}$ & & PG 663 & Th \\
\hline ALSTROEMERIACEAE & Bomarea edulis Herb & $\mathrm{X}$ & $\mathrm{X}$ & PG 425, JRM 505 & $\mathrm{Cr}$ \\
\hline \multirow[t]{2}{*}{ AMARANTHACEAE } & Alternanthera brasiliana (L.) Kuntze & $\mathrm{X}$ & & PG 568 & Th \\
\hline & Gomphrena vaga Mart. & $\mathrm{X}$ & & PG 03 & $\mathrm{Cr}$ \\
\hline \multirow[t]{2}{*}{ AMARYLLIDACEAE } & Habranthus itaobinus Ravenna & & $\mathrm{X}$ & PG 732 & $\mathrm{Cr}$ \\
\hline & Hippeastrum stylosum Herb. & $\mathrm{X}$ & $\mathrm{X}$ & PG 747, 751 & $\mathrm{Cr}$ \\
\hline ANACARDIACEAE & Myracrodruon urundeuva M. Allemão & $\mathrm{X}$ & & PG 737 & $\mathrm{Ph}$ \\
\hline APIACEAE & Spananthe paniculata Jacq. & & $\mathrm{X}$ & PG 428 & Th \\
\hline \multirow[t]{6}{*}{ APOCYNACEAE } & Ditassa oxyphylla Turcz. & $\mathrm{X}$ & & PG 471 & $\mathrm{Ph}$ \\
\hline & $\begin{array}{l}\text { Mandevilla dardanoi M. F. Sales, } \\
\text { Kin.-Gouv. \& A. O. Simões }\end{array}$ & $\mathrm{X}$ & $\mathrm{X}$ & KM15, PG 343 & $\mathrm{Ph}$ \\
\hline & Mandevilla scabra K. Schum. & $\mathrm{X}$ & $\mathrm{X}$ & PG 260, 842 & $\mathrm{Cr}$ \\
\hline & Mandevilla tenuifolia (J. C. Mikan) Woodson & $\mathrm{X}$ & $\mathrm{X}$ & KP 271, KM 19 & $\mathrm{Cr}$ \\
\hline & Marsdenia loniceroides E. Fourn. ${ }^{\mathrm{d}}$ & $\mathrm{X}$ & $\mathrm{X}$ & PG 716,733 & $\mathrm{Ph}$ \\
\hline & Matelea maritima (Jacq.) Woodson & $\mathrm{X}$ & $\mathrm{X}$ & PG 312,525 & $\mathrm{Ph}$ \\
\hline
\end{tabular}


continuation

\begin{tabular}{|c|c|c|c|c|c|}
\hline \multirow{2}{*}{ Family } & \multirow{2}{*}{ Species } & \multicolumn{2}{|c|}{ Locality $^{\mathrm{a}}$} & \multirow{2}{*}{ Voucher ${ }^{\mathrm{b}}$} & \multirow{2}{*}{ Life-forms } \\
\hline & & Gua & $\mathrm{Cab}$ & & \\
\hline & Petalostelma martianum E. Fourn. & $\mathrm{X}$ & & PG 420 & $\mathrm{Ph}$ \\
\hline \multirow[t]{4}{*}{ ARACEAE } & Anthurium affine Schott $^{\mathrm{d}}$ & $\mathrm{X}$ & $\mathrm{X}$ & PG 265, 508 & $\mathrm{Ch}$ \\
\hline & Anthurium gracile Engl. & & $\mathrm{X}$ & PG 269 & $\mathrm{Ph}$ \\
\hline & Philodendron acutatum Schott & $\mathrm{X}$ & & PG 461 & $\mathrm{Ph}$ \\
\hline & $\begin{array}{l}\text { Philodendron leal-costae Mayo \& G. M. } \\
\text { Barroso }\end{array}$ & $\mathrm{X}$ & & PG 763 & $\mathrm{Ph}$ \\
\hline ASPARAGACEAE & Agave sisalana Perrine ex Engelm. & $\mathrm{X}$ & & PG 493 & $\mathrm{HCr}$ \\
\hline \multirow[t]{17}{*}{ ASTERACEAE } & Achyrocline satureioides (Lam.) DC. & & $\mathrm{X}$ & PG 761 & Th \\
\hline & Ageratum sp. & & $\mathrm{X}$ & PG 437 & $\mathrm{Th}$ \\
\hline & Bidens pilosa $\mathrm{L}$. & $\mathrm{X}$ & $\mathrm{X}$ & PG 407, 505 & $\mathrm{Th}$ \\
\hline & Delilia biflora (L.) Kuntze & $\mathrm{X}$ & & PG 460 & $\mathrm{Th}$ \\
\hline & Eclipta alba Hassk. & $\mathrm{X}$ & & PG 573 & $\mathrm{Th}$ \\
\hline & Emilia fosbergii Nicolson & $\mathrm{X}$ & & PG 328 & $\mathrm{Th}$ \\
\hline & Emilia sonchifolia (L.) DC. & & $\mathrm{X}$ & PG 345 & $\mathrm{Th}$ \\
\hline & Erechtites hieraciifolius (L.) Raf. ex DC. & & $\mathrm{X}$ & PG 430 & Th \\
\hline & Eupatoriopsis sp. & $\mathrm{X}$ & & PG 259 & $\mathrm{Th}$ \\
\hline & Eupatorium sp. & $\mathrm{X}$ & & PG 570 & $\mathrm{Ph}$ \\
\hline & Gochnatia lucida (DC. ex Mart.) Cabrera & & $\mathrm{X}$ & PG 348 & $\mathrm{Ph}$ \\
\hline & Helichrysum indicum (L.) Grierson ${ }^{\mathrm{d}}$ & $\mathrm{X}$ & $\mathrm{X}$ & PG 561, 506 & Th \\
\hline & Pithecoseris pacourinoides Mart. ex DC. & $\mathrm{X}$ & & PG 479 & $\mathrm{Th}$ \\
\hline & $\begin{array}{l}\text { Platypodanthera melissifolia (DC.) R. M. } \\
\text { King. \& H. Rob. }{ }^{\text {d }}\end{array}$ & $\mathrm{X}$ & $\mathrm{X}$ & PG 409,363 & Th \\
\hline & Vernonia acutangula Gardner & $\mathrm{X}$ & & PG 408 & Th \\
\hline & Vernonia sp. & & $\mathrm{X}$ & PG 514 & $\mathrm{Th}$ \\
\hline & Asteraceae 1 & $X$ & & PG 378 & Th \\
\hline \multirow[t]{2}{*}{ BEGONIACEAE } & Begonia lealii Brade & $\mathrm{X}$ & & PG 262 & $\mathrm{Ph}$ \\
\hline & Begonia saxicola A. DC. ${ }^{\mathrm{d}}$ & $\mathrm{X}$ & $\mathrm{X}$ & KP 270, PG 524 & $\mathrm{Ph}$ \\
\hline \multirow[t]{3}{*}{ BIGNONIACEAE } & Jacaranda cf. jasminoides (Thunb.) Sandwith & $\mathrm{X}$ & & PG 742 & $\mathrm{Ph}$ \\
\hline & Pyrostegia venusta Miers & $\mathrm{X}$ & & PG 670 & $\mathrm{Ph}$ \\
\hline & $\begin{array}{l}\text { Tabebuia cf. impetiginosa (Mart. ex DC.) } \\
\text { Standl. }\end{array}$ & $\mathrm{X}$ & & PG 671 & $\mathrm{Ph}$ \\
\hline BORAGINACEAE & Cordia globosa Kunth & $\mathrm{X}$ & & PG 834 & $\mathrm{Ph}$ \\
\hline \multirow[t]{10}{*}{ BROMELIACEAE } & $\begin{array}{l}\text { Aechmea leptantha (Harms) Leme \& J. A. } \\
\text { Siqueira }^{\mathrm{d}}\end{array}$ & $\mathrm{X}$ & $\mathrm{X}$ & PG 255,330 & $\mathrm{HCr}$ \\
\hline & Bromelia karatas L. & $\mathrm{X}$ & $\mathrm{X}$ & PG 256, 851 & $\mathrm{HCr}$ \\
\hline & $\begin{array}{l}\text { Encholirium spectabile Mart. ex Schult. \& } \\
\text { Schult.f. }\end{array}$ & $\mathrm{X}$ & $\mathrm{X}$ & PG 725, 661 & $\mathrm{Ch}$ \\
\hline & Hohenbergia catingae Ule & $\mathrm{X}$ & & PG 740 & $\mathrm{HCr}$ \\
\hline & Hohenbergia ridleyi $\mathrm{Mez}$ & $\mathrm{X}$ & $\mathrm{X}$ & PG 754, 734 & $\mathrm{HCr}$ \\
\hline & Orthophytum disjunctum L. B. Sm. ${ }^{d}$ & $\mathrm{X}$ & $\mathrm{X}$ & PG 711, 331 & $\mathrm{HCr}$ \\
\hline & Tillandsia gardneri Lindl. & $\mathrm{X}$ & & PG 744 & $\mathrm{Ph}$ \\
\hline & Tillandsia recurvata (L.) L. & $\mathrm{X}$ & & PG 322 & $\mathrm{Ph}$ \\
\hline & Tillandsia stricta Sol. ex Sims. & $\mathrm{X}$ & & PG 743 & $\mathrm{Ph}$ \\
\hline & Tillandsia usneoides (L.) L. & $\mathrm{X}$ & & PG 317 & $\mathrm{Ph}$ \\
\hline \multirow{5}{*}{ CACTACEAE } & Cereus albicaulis (Britton \& Rose) Luetzelb. ${ }^{\mathrm{d}}$ & $X$ & $\mathrm{X}$ & BSA 83, PG 384 & $\mathrm{Ph}$ \\
\hline & Cereus jamacaru DC. & $\mathrm{X}$ & & PG 852 & $\mathrm{Ph}$ \\
\hline & Pilosocereus pachycladus F. Ritter & $\mathrm{X}$ & $\mathrm{X}$ & PG 753,727 & $\mathrm{Ph}$ \\
\hline & Rhipsalis lindbergiana $\mathrm{K}$. Schum. & $\mathrm{X}$ & $\mathrm{X}$ & $\begin{array}{l}\text { Image database, } \\
\text { PG } 517\end{array}$ & $\mathrm{Ph}$ \\
\hline & Rhipsalis sp. & $\mathrm{X}$ & $\mathrm{X}$ & $\begin{array}{l}\text { Image database, } \\
\text { PG } 518\end{array}$ & $\mathrm{Ph}$ \\
\hline
\end{tabular}




\begin{tabular}{|c|c|c|c|c|c|}
\hline \multirow{2}{*}{ Family } & \multirow{2}{*}{ Species } & \multicolumn{2}{|c|}{ Locality $^{\mathrm{a}}$} & \multirow{2}{*}{ Voucher $^{\mathrm{b}}$} & \multirow{2}{*}{ Life-forms ${ }^{\mathrm{c}}$} \\
\hline & & Gua & $\mathrm{Cab}$ & & \\
\hline CANNABACEAE & Trema micrantha (L.) Blume & $\mathrm{X}$ & $\mathrm{X}$ & PG 382,846 & $\mathrm{Ph}$ \\
\hline CELASTRACEAE & Hippocratea volubilis L. & $\mathrm{X}$ & & PG 668 & $\mathrm{Ph}$ \\
\hline CLEOMACEAE & $\begin{array}{l}\text { Cleome cf. pernambucensis Iltis \& Costa, } \\
\text { Silva }\end{array}$ & & $\mathrm{X}$ & PG 434 & Th \\
\hline CLUSIACEAE & Clusia sp. & $\mathrm{X}$ & $\mathrm{X}$ & PG 875, PG 849 & $\mathrm{Ph}$ \\
\hline \multirow[t]{4}{*}{ COMMELINACEAE } & Callisia repens (Jacq.) L. & $\mathrm{X}$ & & PG 453 & Th \\
\hline & Callisia $\mathrm{sp}$. & & $\mathrm{X}$ & PG 426 & $\mathrm{Th}$ \\
\hline & Commelina obliqua Vahl $^{\mathrm{d}}$ & $\mathrm{X}$ & $\mathrm{X}$ & PG 321, 523 & $\mathrm{Th}$ \\
\hline & Dichorisandra hexandra Standl. & $\mathrm{X}$ & & PG 323 & $\mathrm{Ch}$ \\
\hline \multirow[t]{4}{*}{ CONVOLVULACEAE } & Ipomoea megapotamica Choisy & $\mathrm{X}$ & & PG 463 & $\mathrm{Ph}$ \\
\hline & Ipomoea subincana Meisn. & $\mathrm{X}$ & & PG 324 & $\mathrm{Cr}$ \\
\hline & Ipomoea sp. & & $\mathrm{X}$ & PG 346 & $\mathrm{Cr}$ \\
\hline & $\begin{array}{l}\text { Merremia macrocalyx (Ruiz \& Pav.) } \\
\text { O’Donell }\end{array}$ & $\mathrm{X}$ & $\mathrm{X}$ & PG 451, 440 & $\mathrm{Cr}$ \\
\hline COSTACEAE & Costus spiralis Roscoe & $\mathrm{X}$ & & PG 327 & $\mathrm{Ph}$ \\
\hline \multirow[t]{2}{*}{ CUCURBITACEAE } & Cayaponia sp. & $\mathrm{X}$ & & PG 319 & $\mathrm{Ph}$ \\
\hline & Cyclanthera cf. elegans Cogn. & $\mathrm{X}$ & & PG 370 & $\mathrm{Th}$ \\
\hline \multirow[t]{5}{*}{ CYPERACEAE } & $\begin{array}{l}\text { Bulbostylis scabra (J. Presl \& C. Presl) C. } \\
\text { B. Clarke }\end{array}$ & $\mathrm{X}$ & $\mathrm{X}$ & PG 379, 447 & $\mathrm{Th}$ \\
\hline & Cyperus cuspidatus Kunth ${ }^{\mathrm{d}}$ & $\mathrm{X}$ & $\mathrm{X}$ & PG 567, 542 & $\mathrm{Th}$ \\
\hline & Cyperus uncinulatus Schrad. ex Nees & $\mathrm{X}$ & & PG 455 & $\mathrm{Th}$ \\
\hline & Lipocarpha micrantha (Vahl) G. C.Tucker & & $\mathrm{X}$ & PG 477a & $\mathrm{Th}$ \\
\hline & Scleria interrupta Rich. & & $\mathrm{X}$ & PG 519 & $\mathrm{Th}$ \\
\hline \multirow[t]{2}{*}{ DIOSCOREACEAE } & Dioscorea cf. coronata Hauman & & $\mathrm{X}$ & PG 513 & $\mathrm{Cr}$ \\
\hline & Dioscorea dodecaneura Vell. & $\mathrm{X}$ & & PG 477 & $\mathrm{Cr}$ \\
\hline DROSERACEAE & Drosera montana A. St.-Hil. & & $\mathrm{X}$ & PG 446 & $\mathrm{Th}$ \\
\hline \multirow[t]{2}{*}{ ERIOCAULACEAE } & Paepalanthus lamarckii Kunth & & $\mathrm{X}$ & PG 445 & $\mathrm{Th}$ \\
\hline & Paepalanthus myocephalus Mart. ex Körn. ${ }^{\mathrm{d}}$ & $\mathrm{X}$ & $\mathrm{X}$ & PG 475,441 & $\mathrm{Th}$ \\
\hline \multirow[t]{3}{*}{ ERYTHROXYLACEAE } & Erythroxylum pulchrum A. St.-Hil. & $\mathrm{X}$ & & PG 476 & $\mathrm{Ph}$ \\
\hline & Erythroxylum revolutum Mart. & & $\mathrm{X}$ & PG 361 & $\mathrm{Ph}$ \\
\hline & Erythroxylum suberosum A. St.-Hil. & $\mathrm{X}$ & & PG 728 & $\mathrm{Ph}$ \\
\hline \multirow[t]{12}{*}{ EUPHORBIACEAE } & Acalypha brasiliensis Müll. Arg. & $\mathrm{X}$ & $\mathrm{X}$ & BSA 134,97 & $\mathrm{Ph}$ \\
\hline & Acalypha sp. & $\mathrm{X}$ & & BSA 85 & $\mathrm{Ph}$ \\
\hline & Astraea lobata (L.) Klotzsch & & $\mathrm{X}$ & BSA 98 & $\mathrm{Th}$ \\
\hline & Cnidoscolus urens (L.) Arthur ${ }^{\mathrm{d}}$ & $\mathrm{X}$ & $\mathrm{X}$ & BSA 84, 101 & $\mathrm{Ph}$ \\
\hline & Croton hirtus L' Hér. & & $\mathrm{X}$ & PG 369 & $\mathrm{Th}$ \\
\hline & Croton urticifolius Lam. & $\mathrm{X}$ & $\mathrm{X}$ & BSA 87, 99 & $\mathrm{Ph}$ \\
\hline & Dalechampia scandens L. & $\mathrm{X}$ & & BSA 94 & $\mathrm{HCr}$ \\
\hline & Euphorbia comosa Vell. ${ }^{\mathrm{d}}$ & $\mathrm{X}$ & $\mathrm{X}$ & BSA 91, 102 & $\mathrm{Ph}$ \\
\hline & Euphorbia insulana Vell. ${ }^{\mathrm{d}}$ & $\mathrm{X}$ & $\mathrm{X}$ & BSA 88, 96 & $\mathrm{Ph}$ \\
\hline & Manihot sp. & $\mathrm{X}$ & & PG 750 & $\mathrm{Ph}$ \\
\hline & Romanoa tamnoides (A.Juss.) Radcl.-Sm. & $\mathrm{X}$ & & BSA 132 & $\mathrm{Ch}$ \\
\hline & Sapium argutum Huber & $\mathrm{X}$ & & PG 253 & $\mathrm{Ph}$ \\
\hline \multirow[t]{7}{*}{ FABACEAE } & Cajanus cajan (L.) Millsp. & $\mathrm{X}$ & & JO 22 & $\mathrm{Th}$ \\
\hline & Centrosema virginianum (L.) Benth. & $\mathrm{X}$ & & PG 576 & $\mathrm{Th}$ \\
\hline & Chaetocalyx scandens (L.) Urb. & & $\mathrm{X}$ & PG 662 & $\mathrm{Th}$ \\
\hline & Chamaecrista nictitans Moench & $X$ & $\mathrm{X}$ & PG 738, 516 & $\mathrm{Th}$ \\
\hline & Crotalaria lanceolata E. Mey. & $\mathrm{X}$ & $\mathrm{X}$ & PG 309, 590 & $\mathrm{Th}$ \\
\hline & Desmodium incanum DC. & & $\mathrm{X}$ & PG 336 & $\mathrm{Ph}$ \\
\hline & Hymenaea martiana Hayne & & $X$ & PG 660 & $\mathrm{Ph}$ \\
\hline
\end{tabular}




\begin{tabular}{|c|c|c|c|c|c|}
\hline \multirow{2}{*}{ Family } & \multirow{2}{*}{ Species } & \multicolumn{2}{|c|}{ Locality $^{\mathrm{a}}$} & \multirow{2}{*}{ Voucher $^{\mathrm{b}}$} & \multirow{2}{*}{ Life-forms $^{c}$} \\
\hline & & Gua & $\mathrm{Cab}$ & & \\
\hline & Inga vera Willd. & $\mathrm{X}$ & & PG 767 & $\mathrm{Ph}$ \\
\hline & $\begin{array}{l}\text { Macroptilium bracteatum (Nees \& C. } \\
\text { Mart.) Maréchal \& Baudet }\end{array}$ & $X$ & & PG 856 & $\mathrm{Ph}$ \\
\hline & Mimosa arenosa Poir. & & $\mathrm{X}$ & PG 515 & $\mathrm{Ph}$ \\
\hline & Mimosa ursina Mart. & & $\mathrm{X}$ & PG 520 & $\mathrm{Ph}$ \\
\hline & Piptadenia stipulacea (Benth.) Ducke & $X$ & & EC 311 & $\mathrm{Ph}$ \\
\hline & $\begin{array}{l}\text { Senna aversiflora (Herb.) H. S. Irwin \& } \\
\text { Barneby }\end{array}$ & $\mathrm{X}$ & $\mathrm{X}$ & PG 418, 423 & $\mathrm{Ph}$ \\
\hline & $\begin{array}{l}\text { Senna martiana (Benth.) H. S. Irwin \& } \\
\text { Barneby }\end{array}$ & & $\mathrm{X}$ & PG 332 & $\mathrm{Ph}$ \\
\hline & Senna rizzini H. S. Irwin \& Barneby & $\mathrm{X}$ & & PG 470 & $\mathrm{Ph}$ \\
\hline & Stylosanthes scabra Vogel & & $\mathrm{X}$ & PG 356 & Th \\
\hline & Vigna peduncularis Fawc. \& Rendle & $\mathrm{X}$ & $\mathrm{X}$ & PG 488,591 & Th \\
\hline & Zornia myriadena Benth. & & $\mathrm{X}$ & PG 541 & Th \\
\hline GENTIANACEAE & Schultesia guianensis (Aubl.) Malme ${ }^{\mathrm{d}}$ & & $\mathrm{X}$ & PG 436 & Th \\
\hline \multirow{2}{*}{ GESNERIACEAE } & Paliavana tenuiflora Mansf. $^{\text {d }}$ & $\mathrm{X}$ & $\mathrm{X}$ & PG 264, 340 & $\mathrm{Ph}$ \\
\hline & $\begin{array}{l}\text { Sinningia nordestina Chautems, Baracho \& } \\
\text { Siqueira }\end{array}$ & $\mathrm{X}$ & $\mathrm{X}$ & PG 487, 855 & Th \\
\hline \multirow[t]{5}{*}{ LAMIACEAE } & Hyptis cf. calida Mart. ex Benth. & $\mathrm{X}$ & $\mathrm{X}$ & PG 421, 431 & $\mathrm{Ph}$ \\
\hline & Hyptis pectinata Poit. & $\mathrm{X}$ & & PG 571 & $\mathrm{Ch}$ \\
\hline & Hyptis sidifolia (L’Hér.) Briq. & $\mathrm{X}$ & & PG 569 & $\mathrm{Ch}$ \\
\hline & Marsypianthes chamaedrys Kuntze ${ }^{\mathrm{d}}$ & & $\mathrm{X}$ & PG 354 & Th \\
\hline & Vitex regnelliana Moldenke & $\mathrm{X}$ & $\mathrm{X}$ & PG 739, 731 & $\mathrm{Ph}$ \\
\hline LENTIBULARIACEAE & Utricularia nigrescens Sylvén ${ }^{\mathrm{d}}$ & & $\mathrm{X}$ & PG 448 & Th \\
\hline LOASACEAE & Aosa rupestris (Gardner) Weigend & $\mathrm{X}$ & & PG 325 & Th \\
\hline LORANTHACEAE & Struthanthus sp. & & $\mathrm{X}$ & PG 667 & $\mathrm{Ph}$ \\
\hline \multirow[t]{6}{*}{ MALVACEAE } & Corchorus orinocensis Kunth & & $\mathrm{X}$ & PG 367 & Th \\
\hline & Pavonia aschersoniana Gürke & & $\mathrm{X}$ & PG 509 & $\mathrm{Ph}$ \\
\hline & Pavonia cancellata (L.) Cav. & $\mathrm{X}$ & & PG 462 & $\mathrm{Ph}$ \\
\hline & Sida barclayi Baker f. & & $\mathrm{X}$ & PG 757 & $\mathrm{Ph}$ \\
\hline & Sida linifolia Cav. & & $\mathrm{X}$ & PG 362 & Th \\
\hline & Waltheria indica $\mathrm{L}$. & & $\mathrm{X}$ & PG 512 & Th \\
\hline \multirow[t]{4}{*}{ MELASTOMATACEAE } & Clidemia hirta D. Don. & $\mathrm{X}$ & $\mathrm{X}$ & JO 21, PG 845 & Th \\
\hline & Pterolepis polygonoides Triana $^{\mathrm{d}}$ & & $\mathrm{X}$ & PG 588 & Th \\
\hline & Pterolepis tricothoma (Rottb.) Cogn. & & $\mathrm{X}$ & PG 439a & Th \\
\hline & Tibouchina multiflora Cogn. & $\mathrm{X}$ & $\mathrm{X}$ & PG 466, 587 & $\mathrm{Ph}$ \\
\hline MENISPERMACEAE & Cissampelos sp. & $X$ & $\mathrm{X}$ & PG 371, 854 & $\mathrm{Cr}$ \\
\hline MYRTACEAE & Myrciaria sp. & $\mathrm{X}$ & $\mathrm{X}$ & PG 380, 344 & $\mathrm{Ph}$ \\
\hline ONAGRACEAE & Ludwigia cf. leptocarpa (Nutt.) H. Hara & $X$ & & PG 481 & Th \\
\hline \multirow[t]{9}{*}{ ORCHIDACEAE } & $\begin{array}{l}\text { Acianthera ochreata (Lindl.) Pridgeon \& } \\
\text { Chase }^{\mathrm{d}}\end{array}$ & $\mathrm{X}$ & $\mathrm{X}$ & PG 263, 339 & $\mathrm{Ph}$ \\
\hline & Campylocentrum crassirhizum Hoehne & $\mathrm{X}$ & $\mathrm{X}$ & PG 768,765 & $\mathrm{Ch}$ \\
\hline & Catasetum barbatum (Lindl.) Lindl. & $\mathrm{X}$ & & PG 485 & $\mathrm{Cr}$ \\
\hline & $\begin{array}{l}\text { Cyrtopodium flavum (Nees) Link \& Otto ex } \\
\text { Rchb. }^{\text {d }}\end{array}$ & $\mathrm{X}$ & $\mathrm{X}$ & PG 669, 666 & $\mathrm{Cr}$ \\
\hline & Epidendrum difforme Jacq. & $\mathrm{X}$ & & PG 416 & $\mathrm{Ph}$ \\
\hline & Epidendrum rigidum Jacq. & $\mathrm{X}$ & & PG 417 & $\mathrm{Ph}$ \\
\hline & Habenaria pratensis Rchb.f. & $\mathrm{X}$ & $\mathrm{X}$ & PG 422, 342 & $\mathrm{Cr}$ \\
\hline & Habenaria trifida Kunth & & $\mathrm{X}$ & PG 853 & $\mathrm{Cr}$ \\
\hline & Oeceoclades maculata (Lindl.) Lindl. & $\mathrm{X}$ & & PG 318 & $\mathrm{Cr}$ \\
\hline
\end{tabular}




\begin{tabular}{|c|c|c|c|c|c|}
\hline \multirow{2}{*}{ Family } & \multirow{2}{*}{ Species } & \multicolumn{2}{|c|}{ Locality $^{\mathrm{a}}$} & \multirow{2}{*}{ Voucher $^{\mathrm{b}}$} & \multirow{2}{*}{ Life-forms ${ }^{c}$} \\
\hline & & Gua & $\mathrm{Cab}$ & & \\
\hline & Oncidium barbatum Lindl. & $\mathrm{X}$ & & PG 484 & $\mathrm{Cr}$ \\
\hline & Polystachya estrellensis Rchb. f. & $\mathrm{X}$ & $\mathrm{X}$ & PG 257, 729 & $\mathrm{Cr}$ \\
\hline & Prescottia phleoides Lindl. ${ }^{\mathrm{d}}$ & $\mathrm{X}$ & $\mathrm{X}$ & PG 572, 592 & $\mathrm{Cr}$ \\
\hline & Rodriguezia bahiensis Rchb. f. & $\mathrm{X}$ & & PG 764 & $\mathrm{Cr}$ \\
\hline OROBANCHACEAE & $\begin{array}{l}\text { Alectra aspera (Cham. \& Schltdl.) L. O. } \\
\text { Williams }\end{array}$ & & $\mathrm{X}$ & PG 438 & $\mathrm{Th}$ \\
\hline \multirow[t]{2}{*}{ OXALIDACEAE } & Oxalis cratensis Hook. ${ }^{\mathrm{d}}$ & $\mathrm{X}$ & & PG 320 & $\mathrm{Th}$ \\
\hline & Oxalis hedysarifolia Pohl ex Progel & & $\mathrm{X}$ & PG 334 & $\mathrm{Th}$ \\
\hline PASSIFLORACEAE & Passiflora foetida L. & & $\mathrm{X}$ & PG 760 & Th \\
\hline \multirow[t]{2}{*}{ PHYLLANTHACEAE } & Flueggea flexuosa Müll. Arg. & $\mathrm{X}$ & & BSA 93 & $\mathrm{Ph}$ \\
\hline & Phyllanthus niruri L. & $\mathrm{X}$ & $\mathrm{X}$ & BSA 92, 100 & $\mathrm{Th}$ \\
\hline PHYTOLACCACEAE & Microtea maypurensis G. Don. & & $\mathrm{X}$ & PG 360a & Th \\
\hline \multirow[t]{2}{*}{ PIPERACEAE } & Peperomia blanda Kunth & $\mathrm{X}$ & & PG 411 & Th \\
\hline & Peperomia circinnata Link & $\mathrm{X}$ & & PG 755 & $\mathrm{Th}$ \\
\hline PLANTAGINACEAE & Angelonia pubescens Benth. & & $\mathrm{X}$ & PG 844 & $\mathrm{Th}$ \\
\hline \multirow[t]{8}{*}{ POACEAE } & Anthephora hermaphrodita (L.) Kuntze & $\mathrm{X}$ & & JRM 502 & $\mathrm{Th}$ \\
\hline & Eragrostis rufescens Schrad. ex Schult. & $\mathrm{X}$ & & JRM 500 & $\mathrm{Th}$ \\
\hline & Ichnanthus bambusiflorus Döll & & $\mathrm{X}$ & PG 848 & $\mathrm{Ph}$ \\
\hline & Ichnanthus dasycoleus Tutin & & $\mathrm{X}$ & PG 850 & Th \\
\hline & Lasiacis ligulata Hitchc. \& Chase & $\mathrm{X}$ & & PG 566 & $\mathrm{Th}$ \\
\hline & Melinis minutiflora $\mathrm{P}$. Beauv. & & $\mathrm{X}$ & PG 357 & $\mathrm{Ch}$ \\
\hline & Panicum trichoides Sw. & $\mathrm{X}$ & & PG 468 & $\mathrm{Th}$ \\
\hline & $\begin{array}{l}\text { Schizachyrium brevifolium (Sw.) Nees ex } \\
\text { Büse }\end{array}$ & & $\mathrm{X}$ & PG 589 & Th \\
\hline \multirow[t]{5}{*}{ POLYGALACEAE } & Polygala glochidiata Kunth. & & $\mathrm{X}$ & PG 341 & Th \\
\hline & Polygala paniculata $\mathrm{L} \cdot{ }^{\mathrm{d}}$ & & $\mathrm{X}$ & PG 360 & Th \\
\hline & Polygala spectabilis DC. & & $\mathrm{X}$ & PG 759 & $\mathrm{Th}$ \\
\hline & Polygala violacea Aubl. & & $\mathrm{X}$ & PG 338 & $\mathrm{Th}$ \\
\hline & Securidaca diversifolia (L.) S. F. Blake & $\mathrm{X}$ & & PG 745 & $\mathrm{Ph}$ \\
\hline \multirow[t]{3}{*}{ POLYPODIACEAE } & $\begin{array}{l}\text { Microgramma geminata (Schrad.) R. M. } \\
\text { Tryon \& A. F. Tryon }\end{array}$ & $\mathrm{X}$ & & PG 741 & $\mathrm{Ph}$ \\
\hline & $\begin{array}{l}\text { Microgramma vacciniifolia (Langsd. \& } \\
\text { Fisch.) Copel. }\end{array}$ & $\mathrm{X}$ & & PG 482 & $\mathrm{Ph}$ \\
\hline & Polypodium polypodioides (L.) Watt & $\mathrm{X}$ & & PG 757 & $\mathrm{Ph}$ \\
\hline \multirow[t]{2}{*}{ PORTULACACEAE } & Portulaca elatior Mart. ex Rorhb. ${ }^{\mathrm{d}}$ & $\mathrm{X}$ & $\mathrm{X}$ & PG 705, 659 & $\mathrm{Th}$ \\
\hline & Talinum paniculatum (Jacq.) Gaertn. & $\mathrm{X}$ & & PG 836 & Th \\
\hline PRIMULACEAE & Anagallis minima (L.) E. H. L. Krause ${ }^{\mathrm{d}}$ & $\mathrm{X}$ & $\mathrm{X}$ & PG 574, 543 & $\mathrm{Th}$ \\
\hline PTERIDACEAE & Doryopteris pedata (L.) Fée ${ }^{\mathrm{d}}$ & $\mathrm{X}$ & $\mathrm{X}$ & KP 275, PG 419 & $\mathrm{Ch}$ \\
\hline RHAMNACEAE & Gouania colurnifolia Reissek $^{\mathrm{d}}$ & & $\mathrm{X}$ & PG 377 & $\mathrm{Ph}$ \\
\hline \multirow[t]{7}{*}{ RUBIACEAE } & $\begin{array}{l}\text { Diodella apiculata (Willd. ex Roem. \& } \\
\text { Schult.) Delprete }\end{array}$ & $\mathrm{X}$ & $\mathrm{X}$ & PG 316, 366 & $\mathrm{Th}$ \\
\hline & Emmeorhiza umbellata K. Schum. & $\mathrm{X}$ & $\mathrm{X}$ & PG 564, 511 & $\mathrm{Ph}$ \\
\hline & Manettia cordifolia Mart. & $\mathrm{X}$ & & PG 373 & $\mathrm{Ph}$ \\
\hline & Mitracarpus frigidus K. Schum. & $\mathrm{X}$ & $\mathrm{X}$ & PG 258, 333 & $\mathrm{Ch}$ \\
\hline & Randia armata (Sw.) DC. & $\mathrm{X}$ & & PG 837 & $\mathrm{Ph}$ \\
\hline & Spermacoce alata Aubl. & $\mathrm{X}$ & $\mathrm{X}$ & PG 491, 499 & $\mathrm{Th}$ \\
\hline & Tocoyena cf. formosa K. Schum. & $X$ & $X$ & PG 414, 746 & $\mathrm{Ph}$ \\
\hline \multirow[t]{2}{*}{ RUTACEAE } & Esenbeckia febrifuga A. Juss. & $\mathrm{X}$ & $\mathrm{X}$ & PG 835,358 & $\mathrm{Ph}$ \\
\hline & Fagara rhoifolia Engl. & $\mathrm{X}$ & & PG 416a & $\mathrm{Ph}$ \\
\hline SALICACEAE & Casearia sylvestris Sw. & $\mathrm{X}$ & & PG 839 & $\mathrm{Ph}$ \\
\hline
\end{tabular}




\begin{tabular}{|c|c|c|c|c|c|}
\hline \multirow{2}{*}{ Family } & \multirow{2}{*}{ Species } & \multicolumn{2}{|c|}{ Locality $^{\mathrm{a}}$} & \multirow{2}{*}{ - Voucher ${ }^{\mathrm{b}}$} & \multirow{2}{*}{ Life-forms } \\
\hline & & Gua & $\mathrm{Cab}$ & & \\
\hline \multirow[t]{3}{*}{ SAPINDACEAE } & Cardiospermum halicacabum L. & $\mathrm{X}$ & $\mathrm{X}$ & PG 459, 546 & $\mathrm{Ph}$ \\
\hline & Cupania revoluta Radlk. & $\mathrm{X}$ & $\mathrm{X}$ & PG 375, 349 & $\mathrm{Ph}$ \\
\hline & Paullinia pinnata $\mathrm{L}$. & $\mathrm{X}$ & $\mathrm{X}$ & KP 272, JO 07 & $\mathrm{Ph}$ \\
\hline SAPOTACEAE & Chrysophyllum rufum Mart. & $\mathrm{X}$ & & PG 855 & $\mathrm{Ph}$ \\
\hline SCHIZAEACEAE & Anemia flexuosa (Sav.) Sw. & $\mathrm{X}$ & $\mathrm{X}$ & KP 275a, PG 442 & $\mathrm{Ch}$ \\
\hline SCROPHULARIACEAE & $\begin{array}{l}\text { Ameroglossum pernambucensis Fischer, } \\
\text { Vogel \& Lopes }^{\mathrm{d}}\end{array}$ & $\mathrm{X}$ & & PG 261 & $\mathrm{Ph}$ \\
\hline SELLAGINELACEAE & Selaginella potaroensis Jenman ${ }^{\mathrm{d}}$ & & $\mathrm{X}$ & PG 449 & Th \\
\hline SMILACACEAE & Smilax sp. & $\mathrm{X}$ & & PG 326 & $\mathrm{Cr}$ \\
\hline \multirow[t]{3}{*}{ SOLANACEAE } & Capsicum cf. parvifolium Sendtn. & $\mathrm{X}$ & & PG 664 & $\mathrm{Ph}$ \\
\hline & Schwenckia americana L. $^{\mathrm{d}}$ & & $\mathrm{X}$ & PG 352 & Th \\
\hline & Solanum asperum Rich. & $\mathrm{X}$ & & PG 465 & $\mathrm{Ph}$ \\
\hline TURNERACEAE & Piriqueta sp. & & $\mathrm{X}$ & PG 657 & $\mathrm{Ph}$ \\
\hline URTICACEAE & Pilea hyalina Fenzl ${ }^{\mathrm{d}}$ & $\mathrm{X}$ & $\mathrm{X}$ & PG 489,450 & Th \\
\hline \multirow{2}{*}{ VERBENACEAE } & Lantana camara $\mathrm{L}$. & $\mathrm{X}$ & $\mathrm{X}$ & PG 315,510 & $\mathrm{Ph}$ \\
\hline & Lippia alba (Mill.) N.E. Br. & $\mathrm{X}$ & & PG 577 & $\mathrm{Ph}$ \\
\hline \multirow[t]{2}{*}{ VITACEAE } & Cissus simsiana Schult. \& Schult. f. ${ }^{d}$ & $\mathrm{X}$ & $\mathrm{X}$ & PG 376, 368 & $\mathrm{Ph}$ \\
\hline & Cissus cf. subrhomboidea Planch. & $\mathrm{X}$ & & PG 374 & Th \\
\hline Total & - & 154 & 125 & - & - \\
\hline
\end{tabular}

Amongst the species, three have distributions restricted to rocky outcrops in northeastern Brazil: Mandevilla dardanoi (Apocynaceae), Ameroglossum pernambucensis (Scrophulariaceae), and Pithecoseris pacourinoides (Asteraceae) (Sales et al. 2006, MMA 2007, herbarium data). Only M. dardanoi occured in both studied areas, being the other two exclusively found in Guariba.

Eight species are considered endemic to the Caatinga vegetation: Cordia globosa, Encholirium spectabile, Hohenbergia catingae, Pilosocereus pachycladus, Piptadenia stipulacea, Senna martiana, Senna rizzini, and Senna aversiflora (Giulietti et al. 2002).

Agave sisalana (Henderson 2004), Melinis minutiflora (Arce \& Sano 2001), and Crotalaria lanceolata (Lewis 1987) are invasive alien species. Only C. lanceolata occured in both areas, and A. sisalana and M. minutiflora occured only in Guariba and Cabeça de Velho, respectively.

Structure of vegetation - Overall, 1,792 shrub and herbaceous individuals were found, belonging to 56 taxons (table 2). Amongst them, 712 and 1083 were collected at Guariba and Cabeça de Velho, respectively. Another 155 species were found outside the plots, such as Sinningia nordestina (Gesneriaceae), Bomarea edulis (Alstroemeriaceae), and Encholirium spectabile (Bromeliaceae).
Helichrysum indicum (Asteraceae), Cyperus cuspidatus (Cyperaceae), Paepalanthus spp. (Eriocaulaceae) and Portulaca elatior (Portulacaceae) had the highest relative and absolute densities at Guariba. At Cabeça de Velho, on the other hand, Paepalanthus myocephalus, Paepalanthus spp. (Eriocaulaceae), Utricularia nigrescens Sylvén (Lentibulariaceae) and Selaginella potaroensis (Selaginellaceae) presented the highest relative and absolute densities (table 2, figures 6-7).

Cyrtopodium flavum (Orchidaceae), Orthophytum disjunctum (Bromeliaceae), Bulbostylis scabra (Cyperaceae), Aechmea leptantha (Bromeliaceae) and Poaceae 3 had the highest absolute and relative densities at Guariba. At Cabeça de Velho, A. leptantha, Paliavana tenuiflora (Gesneriaceae), Pterolepis polygonoides (Melastomataceae), P. myocephalus and Euphorbia insulana (Euphorbiaceae) had the highest values for these same parameters (table 2, figures 6-7). The Shannon-Wiener $(\mathrm{H})$ diversity index values were 2.572 and 2.547 nats individual $^{-1}$ for Guariba and for Cabeça de Velho, respectively.

Biological spectrum - At Guariba the phanerophytes represented $49 \%$ (76 spp.) of the total number found, and the therophytes came in second with $28 \%$ (43 spp.). They were followed by the cryptophytes $(12 \%, 18)$, chamaephytes $(6.5 \%, 10)$ and hemicryptophytes $(4.5 \%, 7)$. 
Table 2. Species collected in the plots and their structural attributes, from two crystalline rocky outcrops in the Brazilian northeast semi-arid region: Pedra da Guariba and Pedra Cabeça de Velho. (AD = absolute density; AF = absolute frequency; $\mathrm{RD}=$ relative density; $\mathrm{RF}=$ relative frequency).

\begin{tabular}{|c|c|c|c|c|c|c|c|c|}
\hline \multirow{2}{*}{ Species } & \multicolumn{4}{|c|}{ Pedra da Guariba } & \multicolumn{4}{|c|}{ Pedra Cabeça de Velho } \\
\hline & $\mathrm{AD}$ & $\mathrm{AF}$ & $\mathrm{RD}$ & $\mathrm{RF}$ & $\mathrm{AD}$ & $\mathrm{AF}$ & $\mathrm{RD}$ & RF \\
\hline Acianthera ochreata (Lindl.) Pridgeon \& Chase & 1 & 6.67 & 0.15 & 1.67 & - & - & - & - \\
\hline Aechmea leptantha (Harms) Leme \& J. A. Siqueira & 3 & 20 & 0.42 & 5 & 13 & 66.67 & 1.2 & 12.35 \\
\hline Ameroglossum pernambucensis Fischer, Vogel \& Lopes & 3 & 6.67 & 0.42 & 1.67 & - & - & - & - \\
\hline Anagallis minima (L.) E. H. L. Krause & 26 & 6.67 & 3.65 & 1.67 & - & - & - & - \\
\hline Anthurium affine Schott & 1 & 6.67 & 0.15 & 1.67 & - & - & - & - \\
\hline Asteraceae 1 & - & - & - & - & 2 & 6.67 & 0.19 & 1.24 \\
\hline Asteraceae 2 & - & - & - & - & 4 & 13.33 & 0.37 & 2.47 \\
\hline Begonia saxicola $\mathrm{A} . \mathrm{DC}$. & - & - & - & - & 5 & 20 & 0.46 & 3.7 \\
\hline Bulbostylis scabra (J. Presl \& C. Presl) C. B. Clarke & 17 & 33.33 & 2.39 & 8.33 & 21 & 20 & 1.94 & 3.7 \\
\hline Cereus albicaulis (Britton \& Rose) Luetzelb. & 1 & 6.67 & 0.15 & 1.67 & 1 & 6.67 & 0.09 & 1.24 \\
\hline Cissus simsiana Schult. \& Schult. f. & 1 & 6.67 & 0.15 & 1.67 & - & - & - & - \\
\hline Cnidoscolus urens (L.) Arthur & 6 & 6.67 & 0.84 & 1.67 & 4 & 20 & 0.37 & 3.7 \\
\hline Commelina obliqua Vahl & 12 & 6.67 & 1.68 & 1.67 & - & - & - & - \\
\hline Commelinaceae 1 & 6 & 6.67 & 0.84 & 1.67 & - & - & - & - \\
\hline Commelinaceae 2 & 1 & 13.33 & 0.15 & 3.33 & - & - & - & - \\
\hline Cyperaceae 1 & 3 & 6.67 & 0.42 & 1.67 & - & - & - & - \\
\hline Cyperaceae 2 & 3 & 6.67 & 0.42 & 1.67 & - & - & - & - \\
\hline Cyperaceae 3 & 2 & 6.67 & 0.28 & 1.67 & - & - & - & - \\
\hline Cyperaceae 4 & 1 & 6.67 & 0.15 & 1.67 & - & - & - & - \\
\hline Cyperus cuspidatus Kunth & 141 & 6.67 & 19.8 & 1.67 & - & - & - & - \\
\hline Cyrtopodium flavum (Nees) Link \& Ott & 6 & 6.67 & 0.84 & 1.67 & - & - & - & - \\
\hline Doryopteris pedata (L.) Fée & 2 & 40 & 0.28 & 10 & 14 & 13.33 & 1.3 & 2.47 \\
\hline Euphorbia comosa Vell. & 3 & 6.67 & 0.42 & 1.67 & 1 & 6.67 & 0.09 & 1.24 \\
\hline Euphorbia insulana Vell. & - & - & - & - & 8 & 13.33 & 0.74 & 2.47 \\
\hline Gouania colurnifolia Reissek & 1 & 6.67 & 0.15 & 1.67 & - & - & - & - \\
\hline Helichrysum indicum (L.) Grierson & 155 & 13.33 & 21.77 & 3.33 & - & - & - & - \\
\hline Mandevilla dardanoi M. F. Sales, Kin.-Gouv. \& A. O. Simões & 4 & 6.67 & 0.56 & 1.67 & - & - & - & - \\
\hline Marsdenia loniceroides E. Fourn. & - & - & - & - & 1 & 6.67 & 0.09 & 1.24 \\
\hline Marsypianthes chamaedrys Kuntze & - & - & - & - & 2 & 13.33 & 0.19 & 3.33 \\
\hline Orthophytum disjunctum L. B. Sm. & 5 & 33.33 & 0.7 & 8.33 & 1 & 6.67 & 0.09 & 1.24 \\
\hline Oxalis cratensis Hook. & 1 & - & 0.09 & - & 1 & 6.67 & 0.09 & 1.24 \\
\hline Paepalanthus myocephalus Mart. ex Körn. & 22 & 33.33 & 3.08 & 8.33 & 321 & 6.67 & 29.63 & 1.24 \\
\hline Paepalanthus spp. & 127 & - & 17.83 & - & 171 & 6.67 & 15.79 & 1.24 \\
\hline Paliavana tenuiflora Mansf. & 2 & 13.33 & 0.28 & 3.33 & 12 & 33.34 & 1.11 & 6.18 \\
\hline Pilea hyalina Fenzl & 15 & 6.67 & 2.1 & 1.67 & 54 & 6.67 & 4.99 & 1.24 \\
\hline Platypodanthera melissifolia (DC.) R. M. King. \& H. Rob. & 1 & 13.33 & 0.09 & 3.33 & - & - & - & - \\
\hline Poaceae 1 & 7 & 6.67 & 0.98 & 1.67 & 76 & 20 & 7.02 & 3.7 \\
\hline Poaceae 2 & 3 & 6.67 & 0.42 & 1.67 & 13 & 6.67 & 1.2 & 1.24 \\
\hline Poaceae 3 & 2 & 13.33 & 0.28 & 3.33 & 1 & 6.67 & 0.09 & 1.24 \\
\hline Poaceae 4 & - & 6.67 & - & 1.67 & 1 & 13.33 & 0.09 & 2.47 \\
\hline Polygala pa & - & - & _ & - & 1 & 13.33 & 0.09 & 2.47 \\
\hline Portulaca elatior Mart. ex Rorhb. & 107 & 13.33 & 15.02 & 3.33 & - & - & - & - \\
\hline Prescottia phleoides Lindl. & - & - & - & - & 1 & 6.67 & 0.09 & 1.24 \\
\hline Pterolepis polygonoides Triana & - & 13.33 & - & 3.33 & 45 & - & 4.15 & - \\
\hline Schultesia guianensis (Aubl.) Malme & - & - & - & - & 3 & 6.67 & 0.28 & 1.24 \\
\hline Schwenckia americana L. & - & - & - & - & 42 & 33.33 & 3.88 & 6.18 \\
\hline Seedling 1 & 17 & 6.67 & 2.39 & 1.67 & 9 & 13.33 & 0.83 & 2.47 \\
\hline Seedling 2 & 6 & 6.67 & 0.84 & 1.67 & 1 & 13.33 & 0.09 & 2.47 \\
\hline Seedling 3 & - & - & - & - & 1 & 6.67 & 0.09 & 1.24 \\
\hline Seedling 4 & - & - & 6.67 & 1.24 & 1 & 6.67 & 0.09 & 1.24 \\
\hline Seedling 5 & - & - & - & - & 1 & 6.67 & 0.09 & 1.24 \\
\hline Seedling 6 & - & - & - & - & 1 & 6.67 & 0.09 & 1.24 \\
\hline Seedling 7 & - & - & - & - & 16 & 6.67 & 1.48 & 1.24 \\
\hline Seedling 8 & - & - & - & - & 3 & 6.67 & 0.28 & 1.24 \\
\hline Selaginella & - & - & - & - & 97 & 6.67 & 8.95 & 1.24 \\
\hline Utricularia nigrescens Sylvén & - & - & - & - & 133 & 6.67 & 12.29 & 1.24 \\
\hline
\end{tabular}




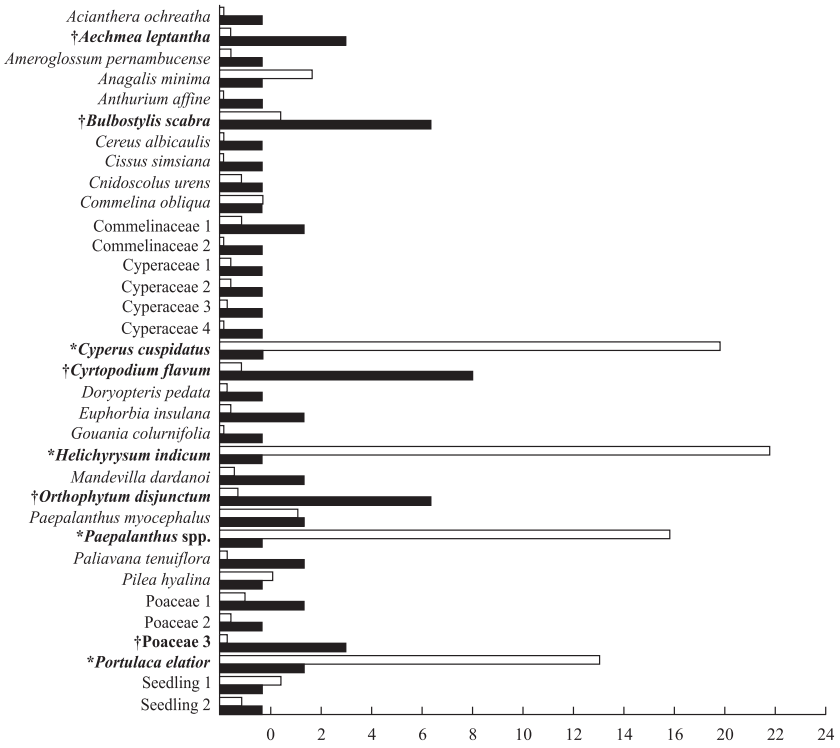

Figure 6. Relative density and relative abundance of species in Pedra da Guariba, a rocky outcrop from semi-arid region of northeastern Brazil. Symbols correspond to (*) species with highest relative densities and $(\dagger)$ species with highest relative frequencies. ( $\square=$ relative density; $\square=$ relative frequency).

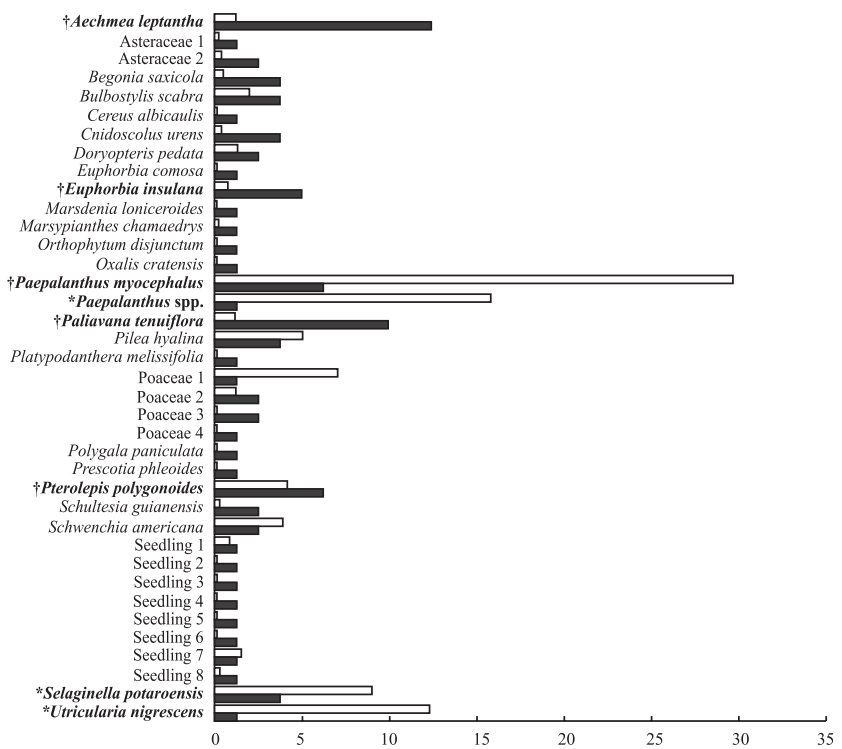

Figure 7. Relative density and relative abundance of species in Pedra Cabeça de Velho, a rocky outcrop from semi-arid region of northeastern Brazil. Symbols correspond to (*) species with highest relative densities and $(\dagger)$ species with highest relative frequencies. ( $\square=$ relative density; $\square=$ relative frequency).

At Cabeça de Velho, the same order was found, except for the smallest difference in the values for phanerophytes $(43 \%, 90 \mathrm{spp}$.) and therophytes $(38 \%, 79)$. The other life forms showed the following values: cryptophytes $(11 \%$, $22)$, chamaephytes $(5 \%, 11)$ and hemicryptophytes $(3 \%, 6)$. See figure 8.

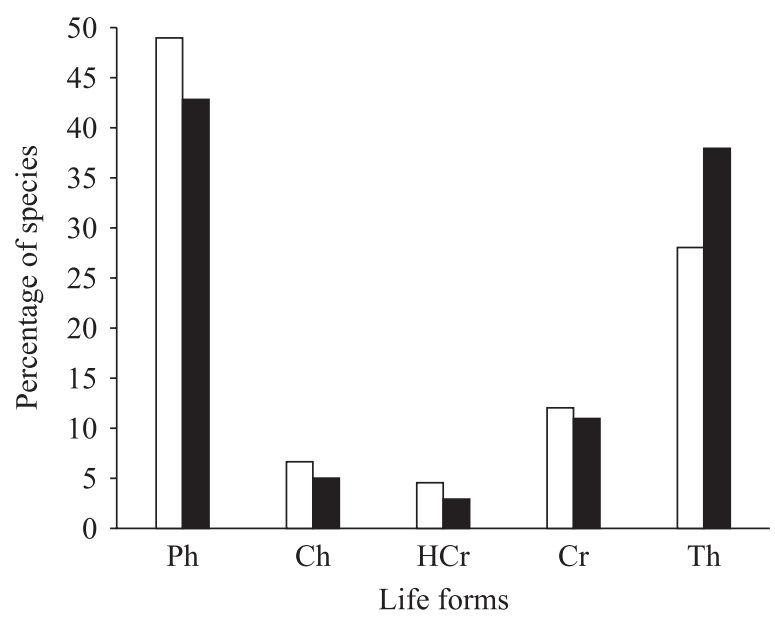

Figure 8. Biological spectrum of two rocky outcrop areas in the semi-arid region of northeastern Brazil: (Gua) Pedra da Guariba and (Cab) Pedra Cabeça de Velho. Life-forms are (Ph) phanerophytes, $(\mathrm{Ch})$ chamaephytes, $(\mathrm{HCr})$ hemicryptophytes (Cr) cryptophytes and(Th) therophytes. ( $\square=$ Pedra da GuaribaGua; = Pedra Cabeça de Velho - Cab).

\section{Discussion}

Floristic - The aforesaid families are very common for rocky outcrops in general (Porembski et al. 1997) and have been found in previous studies in the semi-arid region in Brazil (França et al. 1997, 2005, Araújo et al. 2008, Gomes \& Alves 2009).

Fabaceae and Euphorbiaceae are important components of rock outcrops vegetation in semi-arid region. Chamaecrista, Crotalaria, Senna, Stylosanthes, and Zornia (Fabaceae) and Croton, Cnidoscolus, and Euphorbia (Euphorbiaceae) are also well represented in other northeastern outcrops (França et al. 1997, 2005, 2006, Carneiro-Torres et al. 2002, Araújo et al. 2008, Porto et al. 2008, Gomes \& Alves 2009). These data indicate an important relationship with the caatinga flora, where the families are extremely diversified (Sampaio et al. 2002). However, the current level of knowledge is not enough to score the proportion of species shared by both vegetation types.

Asteraceae, which is the second largest group in the study area, presented low richness in outcrops from northeastern Brazil, except for data found by Araújo et al. (2008). Despite that, some species found in the study areas also occur in other rock outcrops of the region, such as Delilia biflora, Emilia sonchifolia and $P$. pacourinoides, in addition to different species of Gochnatia and Vernonia (França et al. 1997, Araújo et al. 2008, Porto et al. 2008). These taxa differ from those that colonize outcrops from the southeast region 
(e.g. Meirelles et al. 1999, Oliveira \& Godoy 2007), with exception of widely distributed species such as Achyrocline satureioides (e.g. see Ribeiro et al. 2007).

Orchidaceae is well represented in almost all Brazilian rocky outcrops (e.g. França et al. 1997, 2005, Porembski et al. 1997, Gomes \& Alves 2009) as well as in the presently studied areas. Cyrtopodium, Catasetum, and Habenaria are the most widespread genera of this family in the northeastern rock outcrops (França et al. 1997, Araújo et al. 2008, Porto et al. 2008, Gomes \& Alves 2009), and they were also found here.

The richness of Bromeliaceae is also in accordance with other studies of rock outcrops in Brazil (see França et al. 2005, 2006, Caiafa \& Silva 2007, Gomes \& Alves 2009, Ribeiro et al. 2007). Each region is characterized by a restricted group of taxa from this family in generic or infra-generic level and only Tillandsia is common along Brazilian east coast (e.g. Meirelles et al. 1999, Oliveira \& Godoy 2007, França et al. 2006). For this family, $50 \%$ of the species occurred only at Guariba. In other words, a peculiar flora for each region or a differentiated one even in geographically close areas is very common in rock outcrops (Barthlott et al. 1993). Finally, Poaceae, the fifth richest family within the study area, is also one of the most representative families in the outcrops, but at Pantropical scale (see Porembski \& Barthlott 2000).

The two areas have distinct flora, even with elevated Jaccard similatity index (0.347), considering the values of the same similarity index (from 0.05 to 0.25 ) calculated (from 0.05 to 0.25 ) in another study comparing rock outcrops of northeastern Brazil (Gomes \& Alves 2009). The differences in the richness and floristic composition could be related to several factors, such as altitude, historical devastation, and ecological succession (Begon et al. 2006). For example, orographic precipitation is more common in Cabeça de Velho (personal observations) and can help the establishment of more hydrophytic species, such as Schultesia guianensis. This hydrophytic group includes U. nigrescens, and Drosera montana, carnivorous plants that are exclusively found at Cabeça de Velho (table 1). They are cited as being species well adapted to rocky outcrops, indicating a dystrophic substrate (Michelangeli 2000, Saridakis et al. 2004). As a consequence of this nutrient limitation, combined with other edaphic and climatic factors, the plants show morphophysiological adaptations that result in high levels of endemism in these environments (Ibisch et al. 1995, Porembski et al.1996, Biedinger et al. 2000, Seine et al. 2000). Even thought the West face (leeward) of the rock outcrops was not evaluated, its composition appears to be very similar to the one at the East face (windward). However, the richness is apparently lower due to the absence of some mesophyte and hydrophytic species.

Invasive species in the study areas may cause severe damage to the native community due to their high competitiveness (Abe et al. 2008), and their occurrence has already been observed in other rock outcrops (Meirelles et al. 1999, Porembski 2000). Their impact on the rock outcrop native vegetation is still unknown. But, apparently, A. sisalana competes for physical space with Hohenbergia catingae (Bromeliaceae) because both occur in very similar edaphic conditions (personal observations).

Restricted species - Regarding the restrict taxa, the available information on rock outcrops in northeastern Brazil is scarce. Based on Giulliett et al. (2002), Sales et al. (2006), MMA (2007) and on herbarium data (Species Link 2010), only three species can be identified. Among them, $M$. dardano i is very frequent in the study areas and occurs in other rock outcrops of the Borborema Plateau (Porto et al. 2008, Gomes \& Alves 2009, personal observations). Other species of the genus Mandevilla have a close relationship with rocky substrates, and some of them, such as $M$. dardanoi generally occur associated to Bromeliaceae species (Sales et al. 2006).

Ameroglossum pernambucense usually occurs in small cracks. This microhabitats specificity may explain its lower frequency in nature and local herbaria. Until not so long ago, A. pernambucense was known only in the type locality (Fischer et al. 1999, Giulietti et al. 2004), geographically very close to the two studied areas; nowadays, it is known from other outcrops in the states of Pernambuco and Paraíba (Porto et al. 2008, personal observations). The third species, P. pacourinoides, known from more localities than the others, occurs only in rocky substrates, from calcareous at Chapada do Apodi (state of Ceará) to granitics at Borborema Plateau (Species Link 2010).

Structure of vegetation - The Shannon-Wiener diversity index values from both study areas are similar to the value (2.07 nats individual $\left.{ }^{-1}\right)$ for another inselberg in the semiarid region (França et al. 2006). These values are also close to the values found (2.08-2.52 nats individual $\left.{ }^{-1}\right)$ for the herbaceous community in a nearby caatinga area (Araújo et al. 2005).

The vegetation structure analyses showed that many species are rare, because $75 \%$ of the total of 211 were not re-collected in the plots. The majority of these species registered in the plots presented low values for the structural traits in both areas, with few species being 
dominant or frequent. Futhermore, the species with the highest densities and frequencies were diversified between the areas.

The species with the highest density values had proportionally more individuals, yet with lower frequency. Amongst them, only P. myocephalus was found in more than one plot (five) in the area of Cabeça de Velho. The low occurrence of the other species is probably due to the lack of sites with adequate environment conditions. For example, the individuals of $H$. indicum occur exclusively in flooded areas and complete their life cycle in the rainy season.

Aechmea leptantha and O. disjunctum (Bromeliacee) are amongst the most frequent species in the plots and are in accordance with other outcrops in the semi-arid region (França et al. 2006). Aechmea leptantha is important for vegetation islands formation and seems to be the pioneer in the colonization of bare rock patches in the study areas. Encholirium spectabile seems to exert this role in the leeward face, apparently being the dominant species throughout this side. This shows that Bromeliaceae is a taxonomic group with a high importance to the vegetation structure of the local rock outcrops. Paliavana tenuiflora (Gesneriaceae) characterizes the vegetation physiognomy of these outcrops as well. It was the second most frequent species at Cabeça de Velho and one of those which had the sixth highest frequency in Guariba. None of the invasive species were relevant on the vegetation structure.

Biological spectrum - The physical structure of plant communities is also indicated by the species life forms (Philips 1982). In this aspect, the biological spectrum of both areas was similar. Both areas presented a higher proportion of phanerophytes, as well as it occurs in other rock outcrops in Brazil (Meirelles et al. 1999, Conceição \& Giulietti 2002, Ribeiro \& Medina 2002, Conceição \& Pirani 2005, França et al. 2005, 2006, Caiafa \& Silva 2005, Conceição et al. 2007, Ribeiro et al. 2007, Araújo et al. 2008). The phanerophytes are represented in the study areas by different growth forms, such as vines, shrubs and trees, and they occur preferentially in patches with deeper soil. Amongst the phanerophytes, only Begonia saxicola (Begoniaceae) was found in patches with thin humus layer.

Even though the therophytes characterize the studied vegetation, their proportion is variable between them. Therophytes are well represented in rock outcrops in Africa (Porembski et al. 1996, Porembski \& Barthlott 1997). In Brasil, the therophyte group was the most common in only one rock outcrop in the state of Ceara,
(Araújo et al. 2008). Climatic factors, such as seasonal water availability, are described as being the main modulators in the life forms distribution (Raunkiaer 1934). However, under the same mesoclimatic conditions, the two rock outcrops presented distinct proportions of therophytes, and Cabeça de Velho is the only one with recent fire historic. The dominance of therophytes after the fire episodes and its subsequent decrease in later stages of the community is recognized in mediterranean ecosystems (e.g. see Hanes 1971, Verroios $\&$ Georgiadis 2002). This may suggest that the results observed at Cabeça de Velho are a similar reaction from the vegetation to this factor.

The other life forms had a low representativity. The cryptophytes were restricted to the outcrop patches with deeper soils, due to their characteristic underground systems (see Raunkiaer 1934, Galán de Mera et al. 1999). The small proportion of hemicryptophytes found is not in agreement with previous studies carried out in Brazil, where this group is generally well represented (Conceição \& Pirani 2005, Ribeiro \& Medina 2002, França et al. 2005, Conceição et al. 2007, Ribeiro et al. 2007). Differently from the cryptophytes, species of this life form colonize patches of bare rock, as demonstrated by $A$. leptantha. On the other hand, the low occurrence of chamaephytes is in accordance with the data collected from other outcrops of northeastern Brazil (Araújo et al. 2008, Porto et al. 2008), although this life form is more frequent in outcrops of milder climate, in the southeast of the country. (e.g. Ribeiro et al. 2007).

In conclusion, the rock outcrops studied have a floristic relationship with other outcrops of the region and the country, but also have peculiarities in terms of flora and structure. In the biological spectrum, the high proportion of therophytes is the main difference amongst them, but more studies about the possible effects of fire in the life forms succession pattern are required. One very important characteristic is the presence of species from the surrounding vegetation, making the rock outcrops potential sources for the regeneration of this matrix, which is extensively degraded by human activities. Restricted species also aggregate value to the conservation, but more floristic and ecological studies about the semi-arid's rock outcrops vegetation are needed to allow effective inferences about its ecological, phytogeographic and physiognomic relations.

Acknowledgements - The authors thank the following institutions and individuals for their contributions: CNPq for the first author's grant concession; the curators of the herbaria visited; Dr. Katia Torres Ribeiro and Dr. $\mathrm{M}^{\mathrm{a}}$ 
Regina Barbosa for the suggestions on the manuscript; Marcelo Sobral Leite, Basílio Maciel, and Clarissa Gomes for their help in the field work; the members of the MorfoTaxonomia Vegetal laboratory (UFPE) and the specialists A. Alves-Araújo (Amaryllidaceae), A. Rapini (Apocynaceae - Asclepiadoideae), B.S. Amorim (Euphorbiaceae and Malvaceae), D. Amorim (Passifloraceae), E. Córdula (Fabaceae), E. Franklin (Piperaceae), J.R. Maciel (Poaceae), L. Santos Silva (Bignoniaceae), L.P. Félix (Orchidaceae), M.F. Lucena (Euphorbiaceae and Phyllanthaceae), M. Ibrahim (Myrtaceae), M. Kaehler (Bignoniaceae), M.T. Vital (Convolvulaceae), T. Pontes (Araceae), and Y. Melo (Alstroemeriaceae) for their collaboration.

\section{References}

ABE, T., WADA, K. \& NAKAGOSHI, N. 2008. Extinction threats of a narrowly endemic shrub, Stachyurus macrocarpus (Stachyuraceae) in the Ogasawara Islands. Plant Ecology 198:169-183.

AB'SÁBER, A.N. 1974. O domínio morfoclimático semi-árido das caatingas brasileiras. Geomorfologia 43:1-39.

AB'SÁBER, A.N. 2003. Os domínios da Natureza no Brasil: potencialidades paisagísticas. Ateliê Editorial, São Paulo.

ARCE, D. \& SANO, P. 2001. Melinis. In Flora Fanerogâmica do Estado de São Paulo (M.G.L. Wanderley, G.J. Shepherd \& A.M. Giulietti, coords.). Editora Hucitec, São Paulo, v.1, p.165-166.

ALVES, R.J.V. \& KOLBEK, J. 1994. Plant species endemism in savanna vegetation on table mountains (campo rupestre) in Brazil. Vegetatio 113:125-139.

ALVES, R.J.V., CARDIN, L. \& KROPF, M.S. 2007. Angiosperm disjunction "Campos rupestres - restingas": a re-evaluation. Acta Botanica Brasilica 21:675-685.

ANDRADE-LIMA, D. 1981. The caatingas dominium. Revista Brasileira de Botânica 4:149-153.

ANDRADE-LIMA, D. 1982. Present-day forest refuges in northeastern Brazil. In Biological diversification in the tropics. (G.T. Prance, ed.). Columbia University Press, New York, p.247-251.

APG III. 2009. An update of the Angiosperm Phylogeny Group classification for the orders and families of flowering plants: APG III. Botanical Journal of the Linnean Society 161:105-121.

ARAÚJO, E.L., SILVA, K.A., FERRAZ, E.M.N., SAMPAIO, E.V.S.B. \& SILVA, S.I. 2005. Diversidade de herbáceas em microhabitats rochoso, plano e ciliar em uma área de caatinga, Caruaru, PE, Brasil. Acta Botanica Brasilica 19:285-294.

ARAÚJO, F.S., OLIVEIRA, R.F. \& LIMA-VERDE, L.W. 2008. Composição, espectro biológico e síndromes de dispersão da vegetação de um inselbergue no domínio da caatinga, Ceará. Rodriguésia 59:659-671.
BARTHLOTT, W., GRÖGER, A. \& POREMBSKI, S. 1993. Some remarks on the vegetation of tropical inselberg: diversity and ecological differentiation. Biogeographica 69:105-124.

BEGON, M., TOWNSEND, C.R. \& HARPER, J.L. 2006. Ecology: from individuals to ecosystems. Blackwell Publishing Ltd, Oxford.

BIEDINGER, N., POREMBSKI, S. \& BARTHLOTT, W. 2000. Vascular plants on inselberg: vegetative and reproductive strategies. In Inselbergs: biotic diversity of isolated rock outcrops in tropical and temperate regions (Porembski, S. \& Barthlott, W., eds.). Ecological Studies 146, Berlin, p.117-142.

CAIAFA, A.N. \& SILVA, A.F. DA 2005. Composição florística e espectro biológico de um campo de altitude no Parque Estadual da Serra do Brigadeiro, Minas Gerais - Brasil. Rodriguésia 56:163-173.

CAIAFA, A.N. \& SILVA, A.F. DA 2007. Structural analysis of the vegetation on a higland granitic rock outcrop in Southeast Brazil. Revista Brasileira de Botânica 30: 657-664.

CARNEIRO-TORRES, D.S., FRANÇA, F. \& CORDEIRO, I. 2002. A família Euphorbiaceae na flora de inselbergs da região de Milagres, Bahia, Brasil. Boletim de Botânica da Universidade de São Paulo 20:31-47.

CONCEIÇÃO, A.A. \& GIULIETTI, A.M. 2002. Composição florística e aspectos estruturais de campo rupestre em dois platôs do Morro do Pai Inácio, Chapada Diamantina, Bahia, Brasil. Hoehnea:29:37-48.

CONCEIÇÃO, A.A. \& PIRANI, J.R. 2005. Delimitação de habitats em campos rupestres na Chapada Diamantina: substratos, composição florística e aspectos estruturais. Boletim de Botânica da Universidade de São Paulo 23:85-111.

CONCEIÇÃO, A.A., PIRANI, J.R. \& MEIRELLES, S.T. 2007. Floristics, structure and soil of insular vegetation in four quartzite-sandstone outcrops of. Revista Brasileira de Botânica 30:641-656.

CONTI, J.B. 2005. A questão climática do nordeste brasileiro e os processos de desertificação. Revista Brasileira de Climatologia 1:7-14.

FISCHER, E., VOGEL, S. \& LOPES, A.V. 1999. Ameroglossum, a new monotypic genus of Scrophulariaceae - Scrophularioideae from Brazil. Feddes Repertorium 110:529-534.

FRANÇA, F., MELO, E. \& SANTOS, C.C. 1997. Flora de inselbergs da região de Milagres, Bahia, Brasil: I. Caracterização da vegetação e lista de espécies de dois inselbergs. Sitientibus-Série Ciências Biológicas 17:163-184.

FRANÇA, F., MELO, E., SANTOS, A.K. DE A. DOS, MELO, J.G.A. DO N., MARQUES, M., SILVA-FILHO, M.F.B. DA, MORAES, L. \& MACHADO, C. 2005. Estudo ecológico e florístico em ilhas de vegetação de um inselberg no semi-árido da Bahia, Brasil. Hoehnea 32:93-101. 
FRANÇA, F., MELO, E. \& MIRANDA, J.G. 2006. Aspectos da diversidade da vegetação no topo de um inselberg no semi-árido da Bahia, Brasil. Sitientibus 6:30-35.

GALÁN DE MERA, A., HAGEN, M.A. \& VICENTE ORELLANA, J.A. 1999. Aerophyte, a new life form in Raunkiaer's classification? Journal of Vegetation Science 10:65-68.

GIULIETTI, A.M., HARLEY, R.M., QUEIROZ, L.P., BARBOSA, M.R., BOCAGE, A.L. \& FIGUEIREDO, M.A. 2002. Espécies endêmicas da caatinga. In Vegetação e flora da caatinga (E.V.S.B. Sampaio, A.M. Giulietti, J. Virgínio \& C.F.L. Gamarra-Rojas, eds.). Associação de Plantas do Nordeste, Recife, p.103-115.

GIULIETTI, A.M, BOCAGE, A.L. DU, CASTRO, A.A.J.F., GAMARRA-ROJAS, C.F.L., SAMPAIO, E.V.S.B., VIRGÍNIO, J., PAGANUCCI, L, FIGUEIREDO, M.A., RODAL, M.J.N., BARBOSA, M.R.V. \& HARLEY, R. 2004. Diagnóstico da vegetação nativa do bioma Caatinga. In Biodiversidade da Caatinga: áreas e ações prioritárias para a conservação (MMA, UFPE, CI \& CPATSA., orgs.). MMA, Brasilia, p.45-90.

GOMES, P. \& ALVES, M. 2009. Floristic and vegetational aspects of an inselberg in the semi-arid region of Northeast Brazil. Edinburgh Journal of Botany 66:1-18.

HANES, T.L. 1971. Succession after fire in the chaparral of Southern California. Ecological Monographs 41:27-52.

HENDERSON, A. 2004. Agavaceae. In Flowering plants of the Neotropics (N. Smith, S.A. Mori, A. Henderson, D.Wm. Stevenson \& S.V. Heald, eds.). Princeton University Press, Princeton, p.405-406.

IBISCH, P.L., RAUER, G., RUDOLPH, D. \& BARTHLOTT, W. 1995. Floristic, biogeographical and vegetation aspect of Pre-Cambrian rock outcrops (inselberg) in eastern Bolivia. Flora 190:299-314.

ITEP 2010. Instituto de Tecnologia de Pernambuco, Recife, BRA. http://www.itep.br/LAMEPE.asp. (accessed 2010 Sep 16).

LEWIS, G.P. 1987. Legumes of Bahia. Kew Publishing, Londres.

MARTINELLI, G. 2007. Montain biodiversity in Brazil. Revista Brasileira de Botânica 30:587-597.

MEIRELLES, S.T., PIVELLO, V.R. \& JOLY, C.A. 1999. The vegetation of granite rock outcrops in Rio de Janeiro, Brazil, and the need for its protection. Environmental Conservation 26:10-20.

MICHELANGELI, F.A. 2000. Species composition and species-area relationships in vegetation isolates on the summit of a sandstone mountain in southern Venezuela. Journal of Tropical Ecology 16:9-82.

MMA-Ministério do Meio Ambiente. 2007. Áreas prioritárias para conservação, uso sustentável e repartição dos benefícios da biodiversidade brasileira. http: http:// www.mma.gov.br/estruturas/sbf_chm_rbbio/_arquivos/ caatinga_fichas_das_areas_prioritarias.pdf (accessed 2010 Sep 16).
MUELLER-DUMBOIS, D. \& ELLENBERG, H. 1974. Aims and methods of vegetation ecology. John Wiley \& Sons, New York.

OLIVEIRA, R.B. DE \& GODOY, S.A.P. DE 2007. Composição florística dos afloramentos rochosos do Morro do Forno, Altinópolis, São Paulo. Biota Neotropica 7:37-47.

OOSTING, H.J. \& ANDERSON, L.E. 1939. Plant succession on granite rock in Eastern North Carolina. Botanical Gazette 100:750-768.

PHILLIPS, D.L. 1982. Life-forms of outcrop plants. American Midland Naturalist 107:206-208.

POREMBSKI, S. 2000. The invasibility of tropical granite outcrops ('inselbergs') by exotic weeds. Journal of the Royal Society of Western Australia 83: 131-137.

POREMBSKI, S. \& BARTHLOTT, W. 1997. Seasonal dynamics of plant diversity on inselbergs in the Ivory Coast (West Africa). Botanica Acta 110:466-47.

POREMBSKI, S. \& BARTHLOTT, W. 2000. Inselbergs: biotic diversity of isolated rock outcrops in tropical and temperate regions. Ecological Studies 146, Berlin.

POREMBSKI, S., SZARZYNSKI, J., MUND, J.P. \& BARTHLOTT, W. 1996. Biodiversity and vegetation of small-sized inselbergs in a West African rain Forest (Taï, Ivory Coast). Journal of Biogeography 23:47-55.

POREMBSKI, S., SEINE, R. \& BARTHLOTT, W. 1997. Inselberg vegetation and biodiversity of granite outcrops. Journal of the Royal Society of Western Australia 80:193-199.

PORTO, P.A.F., ALMEIDA, A., PESSOA, W.J., TROVÃO, D. \& FELIX, L.P. 2008. Composição florística de um inselbergue no Agreste paraibano, município de Esperança, Nordeste do Brasil. Revista Caatinga 21:214-223.

RAUNKIAER, C. 1934. The life forms of plants and statistical geography. Clarendon, Oxford.

RIBEIRO, K.T. \& MEDINA, B.O. 2002. Estrutura, dinâmica e biogeografia das ilhas de vegetação sobre rocha do Planalto do Itatiaia, RJ. Boletim do Parque Nacional do Itatiaia 10:1-84.

RIBEIRO, K.T., MEDINA, B.M.O. \& SCARANO, F.R. 2007. Species composition and biogeographic relations of the rock outcrop flora on the high plateau of Itatiaia, SE-Brazil. Revista Brasileira de Botânica 30:623-639.

RODAL, M.J.N., SALES, M.F., SILVA, M.J. DA \& SILVA, A.G. DA. 2005. Flora de um Brejo de Altitude na escarpa oriental do planalto da Borborema, PE, Brasil. Acta Botanica Brasilica 19:843-858.

SALES, M.F. DE, KINOSHITA, L.S \& SIMÕES, A.O. 2006. Eight new species of Mandevilla Lindley (Apocynaceae, Apocynoideae) from Brazil. Novon 16: 112-128. 
SAMPAIO, E.V.S.B., GIULETTI, A.M., VIRGÍNIO, J. \& GAMARRA-ROJAS, C.F.L. 2002. Vegetação \& Flora da Caatinga. Associação de Plantas do Nordeste APNE, Recife.

SARIDAKIS, D.P., TOREZAN, J.M.D \&ANDRADE, G. 2004. Microhabitat preferences of six Drosera (Droseraceae) from Tibagi River Basin, Paraná State. Brazilian Archives of Biology and Technology 47:495-501.

SEINE, R., POREMBSKI, S. \& BECKER, U. 2000. Phytogeography. In Inselbergs: biotic diversity of isolated rock outcrops in tropical and temperate regions (Porembski, S. \& Barthlott, W., eds.). Ecological Studies 146, Berlin, p.435-448.

SILVA, F.B.R., RICHÉ, G.R., TONNEAU, J.P., SOUZA NETO, N.C. DE, BRITO, L.T. DE L., CORREIA, R.C., CAVALCANTI, A.C., SILVA, F.H.B.B. DA, SILVA, A.B. DA, ARAÚJO FILHO, J.C. DE \& LEITE, A.P. 1993. Zoneamento agroecológico do Nordeste, diagnóstico do quadro natural e agrossocioeconômico. Embrapa, Petrolina.

SPECIES LINK. 2010. Rede de dados Species Link, BRA. http://splink.cria.org.br/. (accessed 2010 Sep 16).
THIERS, B. 2010. Index Herbariorum: A global directory of public herbaria and associated staff. http://sweetgum. nybg.org/ih/ (accessed 2010 Sep 09).

TRYON, R.M. \& TRYON, A.F. 1982. Ferns and allieds plants with special references to Tropical America. SpringerVerlag, New York.

VASCONCELOS-SOBRINHO, J. 1949. As regiões naturais de Pernambuco, o meio e a civilização. Companhia Editora Americana, Rio de Janeiro.

VELOSO, H.P., FILHO, L.C.O., VAZ, A.M.S.F., LIMA, M.P.M., MARQUETE, R., BRAZÃO, J.E.M., FILHO, A.L.R.R., DIAS, B.F.S., PINTO, G.C.P., MAGNAGO, H., PEREIRA, J.B.S., LIMA, J.C.A., DAMBRÓS, L.A., FURTADO, P.P., KLEIN, R.M., FILGUEIRAS, T.S., BARROS, W.D. \& SILVA, Z.L. 1991. Manual técnico da vegetação brasileira. Fundação Instituto Brasileiro de Geografia e Estatística - IBGE, Rio de Janeiro.

VERROIOS, G. \& GEORGIADIS, T. 2002. Post-fire vegetation succession: the case of aleppo pine (Pinus halepensis Miller) forests of Northern Achaia (Greece). Fresenius Environmental Bulletin 11:186 193. 